\title{
The Carter Constant for Inclined Orbits About a Massive Kerr Black Hole: near-circular, near-polar orbits
}

\author{
P. G. Komorowski* \\ Department of Physics and Astronomy, \\ University of Western Ontario, \\ 1151 Richmond Street, \\ London, Ontario, Canada, N6A $3 \mathrm{~K}^{\eta}$ \\ S. R. Valluri门 \\ Department of Physics and Astronomy and \\ Department of Applied Mathematics, \\ University of Western Ontario, \\ 1151 Richmond Street, \\ London, Ontario, Canada, N6A $3 \mathrm{~K}^{\eta}$ \\ M. Houde田 \\ Department of Physics and Astronomy, \\ University of Western Ontario, \\ 1151 Richmond Street, \\ London, Ontario, Canada, N6A $3 K^{7}$
}




\begin{abstract}
In an extreme mass-ratio binary black hole system, a non-equatorial orbit will list (i.e. increase its angle of inclination, $\iota$ ) as it evolves in Kerr spacetime. The abutment, a set of evolving, near-polar, retrograde orbits, for which the instantaneous Carter constant $(Q)$ is at its maximum value $\left(Q_{X}\right)$ for given values of latus rectum $(\tilde{l})$ and eccentricity $(e)$, has been introduced as a laboratory in which the consistency of $d Q / d t$ with corresponding evolution equations for $d \tilde{l} / d t$ and $d e / d t$ might be tested independently of a specific radiation back-reaction model. To demonstrate the use of the abutment as such a laboratory, a derivation of $d Q / d t$, based only on published formulae for $d \tilde{l} / d t$ and $d e / d t$, was performed for elliptical orbits on the abutment. The resulting expression for $d Q / d t$ matched the published result to the second order in $e$. We believe the abutment is a potentially useful tool for improving the accuracy of evolution equations to higher orders of $e$ and $\tilde{l}^{-1}$.
\end{abstract}

PACS numbers: 04.70.Bw,04.30.Db

*Electronic address: pkomoro@uwo.ca

†Electronic address: valluri@uwo.ca

${ }_{\ddagger}^{\ddagger}$ Electronic address: mhoude2@uwo.ca 


\section{INTRODUCTION}

An extreme mass-ratio binary black hole system (EMRI) is composed of a primary object, which can be a Kerr black hole of mass $M \sim 10^{6}-10^{7}$ solar masses with a spin ${ }^{1} \tilde{S}=|\mathbf{J}| / M^{2}$ (where $\mathbf{J}$ is the spin angular momentum), and an orbiting secondary object of mass $m \sim$ 1 - 10 solar masses. Theoretical models to describe the orbital evolution of the secondary object in various situations have been derived and presented in the literature: circular orbits in the equatorial plane of the primary object [1] 6 , elliptical orbits in the equatorial plane [7-13], and an extensive body of research on circular or elliptical orbits inclined with respect to the equatorial plane [14 31]. Such models are used to generate hypothetical gravitational waveforms $(\mathrm{GW})$, which provide templates for use in the detection of gravitation wave signals by pattern recognition (Punturo et al. [32]). The detection of GW radiation by the Earth-based Laser Interferometer Gravitational Wave Observatory (LIGO) or the Laser Interferometer Space Antenna (LISA) depends fundamentally on the availability of correct templates [13, 33, 34].

Performing direct observations of relativistic effects is an important challenge. The Solar System affords one the opportunity to observe and model the motions of natural and artificial bodies in Kerr spacetime in the weak-field, slow motion limit [35, 36]; and recent measurements of artificial-satellite orbits have produced estimates of the Lense-Thirring precession to an accuracy of $10 \%$ [35]. Further, the discovery of Sagittarius A*, a massive black hole $(\mathrm{MBH})$ of $\sim 4.0 \times 10^{6}$ solar masses, at the centre of our galaxy (see [37- 39] and references therein), offers a new opportunity to study Kerr spacetime by the observation of various stars in inclined, highly elliptical orbits, and by the analysis of their orbital dynamics [37 40]. Relativistic effects are difficult to discern since the orbital periods of the stars are in the tens of years [40], and for orbits that come close to the $\mathrm{MBH}$, tidal disruption is a concern ([40] and see Appendix B in [37]); yet, observation has great potential to aid in the study of Kerr spacetime. In the case of an EMRI, unfortunately, the part played by a theoretician is a fiduciary one; thus, the introduction of tools with which the evolution equations can be tested for consistency is most beneficial: the abutment is one such tool, but it is not intended to replace existing methods.

\footnotetext{
${ }^{1}$ Our use of $\tilde{S}$ for black hole spin arose during our initial studies of the work of Barack and Cutler [24].
} 
The concept of the abutment, a boundary that defines a set of near-polar retrograde orbits, was developed and introduced by P. G. Komorowski in his Doctoral thesis [4] and in a previous work [42] (we shall review the abutment in detail in section II B 1); two uses of the abutment had emerged: first, it suggested a means of testing the consistency of the evolution of the Carter constant of circular orbits $(d Q / d t)$ with respect to that of the latus rectum $(d \tilde{l} / d t)$; and second, it permitted a numerical analysis of the rate of change of the orbital angle of inclination, $\iota$, with respect to $\tilde{l}\left((\partial \iota / \partial \tilde{l})_{\min }\right)$ for circular orbits constrained to evolve along the abutment. In this work we shall extend these uses to orbits of non-zero eccentricity $(0 \leq e \leq 1)$ by testing the consistency of expressions for $d Q / d t$ with expressions for $d \tilde{l} / d t$ and $d e / d t$, and we shall perform an analytical treatment of $\iota$ and the list rate of the same. Further, a physically realistic orbital evolution follows the abutment $\left(Q_{X}\right)$ in only one case, the evolution of an orbit in a Schwarzschild black hole $(\mathrm{SBH})$ system $(\tilde{S}=0)$. We shall now consider the general case of an evolving orbit that intersects the abutment, $Q_{X}$, tangentially at a single point (contact of the first order (see 99 in [43])) as it follows a path defined by $Q_{\text {path }}$. Further, by performing our analysis for elliptical orbits, the abutment becomes a two dimensional surface that defines the maximum value of $Q$ for given values of $e$ and latus rectum, $\tilde{l}=l / M$. Therefore one must view the abutment as a set of contiguous points rather than a path to be followed by an evolving orbit; and it is at these points that the derivatives, $\partial Q_{X} / \partial \tilde{l}$ and $\partial Q_{X} / \partial e$, fix the corresponding slopes of $Q_{\text {path }}$. But as reported in [42], the second-order effect ${ }^{2}$ must be included when working with $\iota$ at the abutment.

In section II we shall analytically derive the formula for $\iota$ for elliptical orbits on the abutment, and thus confirm the result for $(\partial \iota / \partial \tilde{l})_{\min }$ [42], which was derived numerically for circular orbits. In addition, we shall analytically derive $\partial \iota / \partial e$ for elliptical orbits that evolve on the abutment. In section III we shall include the effect of the second derivative of $Q_{\text {path }}$ (i.e. the second-order effect) by introducing reductive ansätze for circular and elliptical orbits, and thus create a more physically realistic model for an evolving orbit at the abutment.

Because our abutment model is independent of any specific radiation back-reaction model, we now have a laboratory that allows us to perform tests of established listing formulae. In

\footnotetext{
${ }^{2}$ When we refer to the second-order effect at the abutment, we refer to the second derivative of $Q_{X}$ with respect to $\tilde{l}$, not to $\tilde{S}^{2}$. See [42] for background discussion.
} 
section IV] we shall demonstrate the usefulness of the abutment in testing the consistency of $d Q / d t$ equations with respect to $d \tilde{l} / d t$ and $d e / d t$ evolution equations, and in calculating $d \iota / d t$ for elliptical orbits of small eccentricity (i.e. near-circular). In section $\mathrm{V}$ we shall conclude our work and recommend directions that warrant further study.

We define $\iota$ to be the maximum polar angle reached by the secondary object in its orbit (see equation (42) in [42]). This definition differs from that used by others (Gair and Glampedakis [27] and Glampedakis, Hughes, and Kennefick [23]); but when performing our analysis to the leading order in $\tilde{S}$, there is no significant difference.

\section{AN ANALYTICAL FORMULA FOR THE ANGLE OF INCLINATION OF AN ELLIPTICAL ORBIT ON THE ABUTMENT}

\section{A. Introduction}

The listing of an inclined elliptical orbit of eccentricity $(e)$ can be described by $\partial \iota / \partial \tilde{l}$ and $\partial \iota / \partial e$, where $\iota$ is the angle of inclination of the orbit and $\tilde{l}$ is its latus rectum normalised with respect to the mass $(M)$ of the Kerr black hole (KBH). A set of essential analytical formulae for the orbital constants of motion has been derived in [42]: the Carter constant at the abutment $\left(Q_{X}\right)$, the orbital energy $(\tilde{E})$, and the quantity, $X=\tilde{L}_{z}-\tilde{S} \tilde{E}$, as well as an analytical formula for $\iota$ in terms of these constants of motion. Numerical analysis yielded an equation for $(\partial \iota / \partial \tilde{l})_{\min }$ for circular orbits:

$$
\begin{aligned}
\left(\frac{\partial \iota}{\partial \tilde{l}}\right)_{\min } & \cong-\left(122.7 \tilde{S}-36 \tilde{S}^{3}\right) \tilde{l}^{-11 / 2}-\left(63 / 2 \tilde{S}+35 / 4 \tilde{S}^{3}\right) \tilde{l}^{-9 / 2} \\
& -15 / 2 \tilde{S} \tilde{l}^{-7 / 2}-9 / 2 \tilde{S} \tilde{l}^{-5 / 2}
\end{aligned}
$$

To verify equation (1) analytically, we shall derive the result to order 3 in $\tilde{S}$ (i.e. $O\left(\tilde{S}^{3}\right)$ ). Observe that equation (1) is a series expansion in terms of $\tilde{l}^{-\frac{1}{2}}$. Further, the series coefficients are themselves series expansions of odd powers of $\tilde{S}$. These are important properties, which we shall confirm and investigate. Equation (1) is not sufficient for understanding the effect of radiation back-reaction on the listing of near-polar orbits; therefore, it is necessary to develop an analytical formula for $\iota$ on the abutment so that a more thorough treatment can be made. We shall review the analytical formulae reported in [42] for elliptical orbits, and develop appropriate expansions of those formulae in terms of $\tilde{S}$. The MacLaurin series 
expansions of the functions $1 /(1+x), \sqrt{1+x}, \arccos (x), \cos (x)$, and $\sin (x)$ are essential for this work.

\section{B. Review of Analytical Formulae}

1. The abutment, $Q_{X}$

The analytical formula for $X_{ \pm}^{2}$ (where $\left.X=\tilde{L}_{z}-\tilde{S} \tilde{E}\right)$ for elliptical and inclined orbits about a $\mathrm{KBH}$ was found to be [42]:

$$
X_{ \pm}^{2}=\frac{Z_{5}+Z_{6} Q \pm 2 \tilde{S} \sqrt{Z_{7} Z_{8} Z_{9}}}{\tilde{l}\left(\tilde{l}\left(3-\tilde{l}+e^{2}\right)^{2}-4 \tilde{S}^{2}\left(1-e^{2}\right)^{2}\right)}
$$

where

$$
\begin{gathered}
Z_{5}=\tilde{l}^{3}\left\{\left(\tilde{l}+3 e^{2}+1\right) \tilde{S}^{2}-\tilde{l}\left(3-\tilde{l}+e^{2}\right)\right\} \\
Z_{6}=-2\left(1-e^{2}\right)^{2} \tilde{S}^{4}+2 \tilde{l}\left(2 e^{4}+(2-\tilde{l}) e^{2}+4-\tilde{l}\right) \tilde{S}^{2}-\tilde{l}^{2}\left(3-\tilde{l}+e^{2}\right)^{2} \\
Z_{7}=\tilde{S}^{2}(1+e)^{2}+\tilde{l}(\tilde{l}-2(1+e)) \\
Z_{8}=\tilde{S}^{2}(1-e)^{2}+\tilde{l}(\tilde{l}-2(1-e))
\end{gathered}
$$

and

$$
Z_{9}=\left(\tilde{l}^{5}+\tilde{S}^{2} Q^{2}\left(1-e^{2}\right)^{2}+Q \tilde{l}^{3}\left(3-\tilde{l}+e^{2}\right)\right)
$$

Intriguingly, the roots of $Z_{7}=0$ correspond to the coordinate singularities associated with the event horizon of the $\mathrm{KBH}$, multiplied by $1+e$; for $Z_{8}=0$ the multiplier is $1-e$. The abutment, which lies outside the event horizon, corresponds to a set of orbits for which $Z_{9}=0$ [42], i.e.

$$
\tilde{l}^{5}+\tilde{S}^{2} Q^{2}\left(1-e^{2}\right)^{2}+Q \tilde{l}^{3}\left(3-\tilde{l}+e^{2}\right)=0
$$

The solution of equation $(8)$ is:

$$
Q_{X}=\frac{\tilde{l}^{2}}{2 \tilde{S}^{2}\left(1-e^{2}\right)^{2}}\left(\tilde{l}\left(\tilde{l}-e^{2}-3\right) \pm \sqrt{\tilde{l}^{2}\left(\tilde{l}-e^{2}-3\right)^{2}-4 \tilde{l}\left(1-e^{2}\right)^{2} \tilde{S}^{2}}\right)
$$

Where the minus solution must be taken since the plus solution has a singularity at $\tilde{S}=0$ (unphysical for an $\mathrm{SBH}$ ) and at $e=1$. Further, the value of $Q$ of an evolving orbit cannot 
exceed $Q_{X}$; equation 2 would yield a complex result for $X_{ \pm}^{2}$. Hence $\tilde{L}_{z}$ and $\tilde{E}$ would possess unphysical values.

By performing an expansion in terms of $\tilde{S}^{2}$ one obtains:

$$
Q_{X} \cong \frac{\tilde{l}^{2}}{\left(\tilde{l}-e^{2}-3\right)}+\frac{\tilde{l}\left(1-e^{2}\right)^{2} \tilde{S}^{2}}{\left(\tilde{l}-e^{2}-3\right)^{3}}+2 \frac{\left(1-e^{2}\right)^{4} \tilde{S}^{4}}{\left(\tilde{l}-e^{2}-3\right)^{5}} \ldots
$$

Therefore $Q_{X}=O\left(\tilde{S}^{0}\right)$ and the $j^{\text {th }}$ term of $Q_{X}=O\left(\tilde{S}^{2 j}\right)$. The expansion of $Q_{X}$ in terms of $\tilde{l}$ can be derived from equation 10 once it has been determined to which power of $\tilde{S}$ one wishes to work. This result, and its derivatives with respect to $\tilde{l}$ and $e$, are presented in Appendix A3 for use in our analysis in section IV A.

We return to equation (2). The terms under the square root can be excluded since $Z_{9}=0$. Substitution of $Q_{X}$ into the remaining part of the equation yields:

$$
\begin{aligned}
X_{ \pm}^{2} \cong \tilde{S}^{2}\left(\frac{\tilde{l}\left(\tilde{l}^{2}-4 \tilde{l}-4 e^{2}+4\right)}{\left(\tilde{l}-e^{2}-3\right)^{3}}\right. & \\
& +2 \frac{\left(2-10 e^{2}+e^{2} \tilde{l}-3 \tilde{l}+\tilde{l}^{2}\right)\left(1-e^{2}\right)^{2} \tilde{S}^{2}}{\left(\tilde{l}-e^{2}-3\right)^{5}} \\
& \left.+\frac{\left(6 \tilde{l}^{2}+\left(8 e^{2}-16\right) \tilde{l}+9-74 e^{2}+e^{4}\right)\left(1-e^{2}\right)^{4} \tilde{S}^{4}}{\tilde{l}\left(\tilde{l}-e^{2}-3\right)^{7}}\right) .
\end{aligned}
$$

From equation 11 one finds that $X_{ \pm}^{2}=O\left(\tilde{S}^{2}, \tilde{l}^{0}\right)$. Further analysis yields the result:

$$
\begin{aligned}
X & = \pm \tilde{S} \sqrt{\frac{\tilde{l}\left(\tilde{l}^{2}-4 \tilde{l}-4 e^{2}+4\right)}{\left(\tilde{l}-e^{2}-3\right)^{3}}} \\
& \times\left(1+\frac{\left(1-e^{2}\right)^{2} \tilde{S}^{2}}{\left(\tilde{l}-e^{2}-3\right)^{2} \tilde{l}\left(\tilde{l}^{2}-4 \tilde{l}-4 e^{2}+4\right)} P_{1}\right. \\
& \left.+\frac{1}{2} \frac{\left(1-e^{2}\right)^{4} \tilde{S}^{4}}{\left(\tilde{l}-e^{2}-3\right)^{4} \tilde{l}^{2}\left(\tilde{l}^{2}-4 \tilde{l}-4 e^{2}+4\right)^{2}} P_{2}\right)
\end{aligned}
$$


where

$$
\begin{aligned}
P_{1} & =\tilde{l}^{2}-\left(3-e^{2}\right) \tilde{l}+2-10 e^{2} \\
P_{2} & =5 \tilde{l}^{4}-\left(34-6 e^{2}\right) \tilde{l}^{3}+\left(84-104 e^{2}\right) \tilde{l}^{2} \\
& -\left(88-328 e^{2}+16 e^{4}\right) \tilde{l}+32-292 e^{2}+200 e^{4}-4 e^{6} .
\end{aligned}
$$

\section{Orbital energy, $\tilde{E}$}

The formula for orbital energy, $\tilde{E}$, for inclined elliptical orbits (see equation (44) in [42]) is presented here in a form that more clearly shows that $\tilde{E}=O\left(\tilde{S}^{0}\right)$ :

$$
\tilde{E}=\sqrt{1-\left(1-e^{2}\right) \frac{\tilde{l}^{3}-Q\left(\tilde{l}-\tilde{S}^{2}\right)\left(1-e^{2}\right)-\tilde{l} X^{2}\left(1-e^{2}\right)}{\tilde{l}^{4}}} ;
$$

further, substitution of $Q_{X}(\tilde{S})$ and $X_{ \pm}^{2}(\tilde{S})$ into equation 13 yields an expression for $\tilde{E}$, which can be used directly in our analysis, or in the following form:

$$
\begin{aligned}
\tilde{S}^{2}\left(1-\tilde{E}^{2}\right) & =\frac{\left(1-e^{2}\right)(\tilde{l}-4) \tilde{S}^{2}}{\tilde{l}\left(\tilde{l}-e^{2}-3\right)}-2 \frac{\left(1-e^{2}\right)^{2}\left(e^{2} \tilde{l}+\tilde{l}-6 e^{2}-2\right) \tilde{S}^{4}}{\tilde{l}^{2}\left(\tilde{l}-e^{2}-3\right)^{3}} \\
& -\frac{\left(1-e^{2}\right)^{4}\left(-3-30 e^{2}+4 e^{2} \tilde{l}+\tilde{l}^{2}+e^{4}\right) \tilde{S}^{6}}{\tilde{l}^{3}\left(\tilde{l}-e^{2}-3\right)^{5}} .
\end{aligned}
$$

\section{Orbital angle of inclination, ८}

The exact formula for $\iota$ was derived in Boyer-Lindquist coordinates (BL coordinates) and found to be:

$$
\sin ^{2}(\iota)=\frac{Q+\tilde{L}_{z}^{2}+\tilde{S}^{2}\left(1-\tilde{E}^{2}\right)-\sqrt{\left(Q+\tilde{L}_{z}^{2}+\tilde{S}^{2}\left(1-\tilde{E}^{2}\right)\right)^{2}-4 Q \tilde{S}^{2}\left(1-\tilde{E}^{2}\right)}}{2 \tilde{S}^{2}\left(1-\tilde{E}^{2}\right)},
$$

which suggests an approximate expansion in the cases of small $\tilde{S}$ or for $\tilde{l} \rightarrow \infty$ (for which $\tilde{E} \rightarrow 1$ ) [42. In particular, near-equatorial orbits can also be approximated by such an expansion since $Q \gtrsim 0$. But we are studying near-polar orbits, for which $Q>12$; so it is 
advantageous to exploit the fact that $\tilde{L}_{z} \cong 0$ and convert equation 15 to an alternative form which can be expanded as a series (not strictly in powers of $\tilde{S}^{2}$ ) to obtain:

$$
\begin{aligned}
\cos ^{2}(\iota) & \cong \frac{\tilde{L}_{z}^{2}}{Q+\tilde{L}_{z}^{2}-\tilde{S}^{2}\left(1-\tilde{E}^{2}\right)}-\frac{\tilde{L}_{z}^{4}\left(1-\tilde{E}^{2}\right) \tilde{S}^{2}}{\left(Q+\tilde{L}_{z}^{2}-\tilde{S}^{2}\left(1-\tilde{E}^{2}\right)\right)^{3}} \\
& +\frac{2 \tilde{L}_{z}^{6}\left(1-\tilde{E}^{2}\right)^{2} \tilde{S}^{4}}{\left(Q+\tilde{L}_{z}^{2}-\tilde{S}^{2}\left(1-\tilde{E}^{2}\right)\right)^{5}}
\end{aligned}
$$

It is essential to establish the lowest order of $\tilde{S}$ for each term of equation (16); the results in equations 10 and 11 , and equation 14 to $O\left(\tilde{S}^{2}\right)$ will help.

It was found that $X_{ \pm}=-\sqrt{X_{ \pm}^{2}}$ in the vicinity of the abutment (see section 3.5 in Komorowski et al. [42]); therefore,

$$
\tilde{L}_{z}=-\sqrt{X_{ \pm}^{2}}+\tilde{S} \tilde{E}
$$

Each of the expressions in equations (12) and (13), when expanded as a power series in $\tilde{l}^{-1}$, will have a leading factor of $\tilde{S}$ and unity, respectively. In evaluating equation (17), the leading terms subtract out; therefore, we find that $\tilde{L}_{z}^{2}=O\left(\tilde{S}^{2}, \tilde{l}^{-2}\right)$. The inverse dependence of $\tilde{L}_{z}^{2}$ on $\tilde{l}$ is consistent with the physical meaning of $\tilde{L}_{z}$ for orbits on the abutment. Further, equation 10 indicates that $Q_{X}=O\left(\tilde{S}^{0}, \tilde{l}\right)$; therefore, the first term in equation 16 is $O\left(\tilde{S}^{2}\right)$, and the second term, $O\left(\tilde{S}^{6}\right)$, with each term containing higher order terms of $\tilde{S}$ in increments of 4 .

Taking the square root of both sides of equation $(16)$ yields,

$$
\cos (\iota)=\overbrace{O(\tilde{S})}^{\text {term }} \begin{gathered}
1^{\text {st }} \\
\text { term } \\
\text { term } \\
\left(\tilde{S}^{5}\right)
\end{gathered}+\overbrace{O\left(\tilde{S}^{9}\right)}^{3^{\text {terd }}}
$$

with higher order terms of odd power of $\tilde{S}$. The second term in equation 16 will contribute to equation 18 a factor $O\left(\tilde{S}^{5}\right)$; therefore, to derive an analytical formula for $(\partial \iota / \partial \tilde{l})_{\min }$ valid to $O\left(\tilde{S}^{3}\right)$ (see equation $(1)$ ) it is sufficient to use the first term of equation 16 . If we choose to work in stronger gravitational fields, for which terms of greater order in $\widetilde{S}$ are required, then the second and possibly higher order terms in equation (16) would be used. But we wish to work with terms that contain $\tilde{S}$ and $\tilde{S}^{3}$, to the exclusion of those with $\tilde{S}^{5}$, so we shall restrict our analysis to the first term of the series in equation (16); after taking 
the square root, it can be simplified to yield:

$$
\cos (\iota) \cong \frac{\tilde{L}_{z}}{\sqrt{Q}}\left[1-\frac{1}{2} \frac{\tilde{L}_{z}^{2}}{Q}+\frac{1}{2} \frac{\tilde{S}^{2}\left(1-\tilde{E}^{2}\right)}{Q}\right]
$$

Given $x=\cos (\iota)$, one may calculate $\iota$ to $O\left(\tilde{S}^{3}\right)$ by using the MacLaurin series for $\arccos (x)$ to $O\left(x^{3}\right)$.

\section{Analytical Formula for $\iota(e, \tilde{l})$ on the Abutment}

We shall now evaluate equation $(19)$ analytically by working with the constituent terms as series expansions in $\tilde{S}$, the coefficients of which are expressed in terms of $e$ and $\tilde{l}$; the result to third order in $\tilde{S}$ is our target. An apercu of the method by which the expression in equation 19 is treated appears in Appendix B.

\section{First-order in $\tilde{S}$}

To perform our calculation of $\iota$ to $O(\tilde{S})$ (see Appendix B 1 it is sufficient to use:

$$
\stackrel{(1)}{\iota}=\frac{\pi}{2}-\frac{\stackrel{(1)}{L}_{z}}{\sqrt{Q_{X}}}
$$

where

$$
\frac{\tilde{L}_{z}}{\sqrt{Q_{X}}}=-\tilde{S}\left(e^{2}+3\right)\left(\frac{1}{\tilde{l}^{3 / 2}}+\frac{\left(1+e^{2}\right)}{\tilde{l}^{5 / 2}}+\frac{\left(3+2 e^{2}+e^{4}\right)}{\tilde{l}^{7 / 2}}+\frac{\left(9+5 e^{2}+5 e^{4}+e^{6}\right)}{\tilde{l}^{9 / 2}}\right)
$$

and the number in parenthesis indicates the order in $\tilde{S}$ of the term below it.

\section{Third-order in $\tilde{S}$}

Our third-order equations are more complicated. Consider the third-order equation for $\iota$ :

$$
\stackrel{(3)}{\iota}=\frac{\pi}{2}-x-\frac{1}{6} x^{3}
$$


where

$$
x=\frac{\stackrel{(3)}{L}_{z}}{\sqrt{Q_{X}}}\left(1-\frac{1}{2}\left(\frac{\tilde{L}_{z}}{\sqrt{Q_{X}}}\right)^{2}+\frac{1}{2} \frac{\tilde{S}^{2}\left(1-\tilde{E}^{2}\right)}{Q_{X}}\right)
$$

in which

$$
\frac{\tilde{L}_{z}}{\sqrt{Q_{X}}}=\frac{(1)}{\sqrt{Q_{X}}}-\tilde{S}^{3}\left(1-e^{2}\right)^{2}\left(\frac{1}{\tilde{l}^{7 / 2}}+\frac{1}{2} \frac{11+5 e^{2}}{\tilde{l}^{9 / 2}}\right)
$$

and

$$
\frac{\tilde{S}^{2}\left(1-\tilde{E}^{2}\right)}{Q_{X}}=\left(1-e^{2}\right)\left(\frac{1}{\tilde{l}^{2}}-\frac{4}{\tilde{l}^{3}}\right) \tilde{S}^{2}
$$

(see Appendix B 2. We evaluate equation 22 to obtain the final result, of $O\left(\tilde{S}^{3}\right)$ :

$$
\begin{aligned}
\stackrel{(3)}{\iota} & =\left[\tilde{S}^{3}\left(-8-13 e^{2}-2 e^{4}+5 / 3 e^{6}\right)+\left(e^{2}+3\right)\left(9+5 e^{2}+5 e^{4}+e^{6}\right) \tilde{S}\right] \tilde{l}^{-9 / 2} \\
& +\left[1 / 2\left(1-e^{2}\right)\left(5-e^{2}\right) \tilde{S}^{3}+\left(e^{2}+3\right)\left(3+2 e^{2}+e^{4}\right) \tilde{S}\right] \tilde{l}^{-7 / 2} \\
& +\tilde{S}\left(3+e^{2}\right)\left(1+e^{2}\right) \tilde{l}^{-5 / 2}+\tilde{S}\left(3+e^{2}\right) \tilde{l}^{-3 / 2}+\frac{\pi}{2}
\end{aligned}
$$

\section{Derivatives of $\iota(e, \tilde{l})$ on the Abutment}

By taking the partial derivative of $\iota$ with respect to $\tilde{l}$ (using equation 26) ) one obtains:

$$
\begin{aligned}
\left(\frac{\partial \iota}{\partial \tilde{l}}\right)_{\min } & =-\frac{3}{2}\left[3\left(e^{2}+3\right)\left(9+5 e^{2}+5 e^{4}+e^{6}\right) \tilde{S}-\left(24+39 e^{2}+6 e^{4}-5 e^{6}\right) \tilde{S}^{3}\right] \tilde{l}^{-11 / 2} \\
& -\frac{7}{2}\left[\left(e^{2}+3\right)\left(3+2 e^{2}+e^{4}\right) \tilde{S}+\frac{1}{2}\left(1-e^{2}\right)\left(5-e^{2}\right) \tilde{S}^{3}\right] \tilde{l}^{-9 / 2} \\
& -\frac{5}{2}\left(3+e^{2}\right)\left(1+e^{2}\right) \tilde{S} \tilde{l}^{-7 / 2}-\frac{3}{2}\left(3+e^{2}\right) \tilde{S} \tilde{l}^{-5 / 2}
\end{aligned}
$$

The partial derivative of $\iota$ with respect to $e$ can also be derived:

$$
\begin{aligned}
\left(\frac{\partial \iota}{\partial e}\right)_{\min } & =2 e\left(4\left(6+10 e^{2}+6 e^{4}+e^{6}\right) \tilde{S}-\left(13-5 e^{4}+4 e^{2}\right) \tilde{S}^{3}\right) \tilde{l}^{-9 / 2} \\
& +2 e\left(\left(9+10 e^{2}+3 e^{4}\right) \tilde{S}-\left(3-e^{2}\right) \tilde{S}^{3}\right) \tilde{l}^{-7 / 2} \\
& +4 e\left(2+e^{2}\right) \tilde{S} \tilde{l}^{-5 / 2}+2 e \tilde{S} \tilde{l}^{-3 / 2}
\end{aligned}
$$


The formula in equation (27), when evaluated at $e=0$, matches the numerical result in equation $(1)$ for all of the terms with the exception of $-122.7 \tilde{S} \tilde{l}^{-11 / 2}$, which differs slightly from the analytical result of $-243 / 2 \tilde{S} \tilde{l}^{-11 / 2}$.

\section{E. Directional Derivatives in the $\tilde{l}-e$ Plane}

Consider the constant of motion, $Q$, and the corresponding quantity, $\iota$, in the $\tilde{l}-e$ plane; by using the concept of the directional derivative for two variables, one may represent $d Q / d t$ by the equation:

$$
\frac{d Q}{d t}=\frac{\partial Q}{\partial \tilde{l}} \frac{d \tilde{l}}{d t}+\frac{\partial Q}{\partial e} \frac{d e}{d t}
$$

and in a similar manner we may define,

$$
\frac{d \iota}{d t}=\frac{\partial \iota}{\partial \tilde{l}} \frac{d \tilde{l}}{d t}+\frac{\partial \iota}{\partial e} \frac{d e}{d t}
$$

where the terms $d \tilde{l} / d t$ and $d e / d t$ denote the evolution of $\tilde{l}$ and $e$ to arbitrary order. We have the benefit of knowing the analytical expressions $\partial Q / \partial \tilde{l}$ (see equation (A10) and $\partial Q / \partial e$ (see equation A11) at the abutment, which we can derive to the required order.

A weak-field solution for $d \iota / d t$, in terms of $\tilde{l}$ and $e$, has been derived and reported in the literature (see equation (15a) in [17]):

$$
\frac{d \iota}{d t}=\frac{m \tilde{S}}{M^{2}} \tilde{l}^{-\frac{11}{2}}\left(1-e^{2}\right)^{\frac{3}{2}} \sin (\iota)\left(\frac{244}{15}+\frac{252}{5} e^{2}+\frac{19}{2} e^{4}-\cos \left(2 \psi_{0}\right)\left(8 e^{2}+\frac{26}{5} e^{4}\right)\right),
$$

where the term $\cos \left(2 \psi_{0}\right)$, in which $\psi_{0}$ represents the orientation of the elliptical orbit in the orbital plane, typically averages to zero with the possible exception where the orbit has a large value of $e<1$ [17]. More recently, a solution for $d \iota / d t$ to higher order in $\tilde{l}^{-1}$ (we present the weak-field portion here) was derived by Flanagan and Hinderer [30]:

$$
\frac{d \iota}{d t}=\frac{m \tilde{S}}{M^{2}} \tilde{l}^{-\frac{11}{2}}\left(1-e^{2}\right)^{\frac{3}{2}} \sin (\iota)\left(\frac{266}{15}+\frac{184}{5} e^{2}+\frac{151}{20} e^{4}+\cos (2 \iota)\left(\frac{22}{15}-\frac{62}{5} e^{2}-\frac{39}{20} e^{4}\right)\right),
$$

in which they confirmed a weak-field correspondence to equation (31). In addition to the $\sin (\iota)$ contribution found in both equation 31 and $(32)$, there is a $\cos (2 \iota)$ term in the latter expression.

The trigonometric quantities, $\sin (\iota)$ and $\cos (\iota)$, do not occur in our expressions for $\iota$ and its derivatives at the abutment. But such trigonometric terms are found, usually in 
a product with $\tilde{S}$, in the general evolution equations (i.e. $d \tilde{l} / d t, d e / d t, d Q / d t$, and $d \iota / d t$ ) published in the literature [24, 29, 31]. One may use equations (20) and (21) to derive approximations of $\sin (\iota)$ and $\cos (\iota)$ suitable for working in the leading order of $\tilde{S}$. Further, we may use the approximation of $\cos (2 \iota)$ to corroborate the conclusion that equation (32) is the same as (31) in the weak-field regime. These trigonometric approximations are only valid on the abutment; thus, if it is necessary to perform a differentiation of a trigonometric term (as in equation (A14)), then the differentiation must be performed before making the approximation. Such limitations notwithstanding, the trigonometric approximations are of value to us investigators since they afford us a systematic method for their treatment.

\section{CORRECTION OF $\partial \iota / \partial \tilde{l}$ AND $\partial \iota / \partial e$ FOR SECOND-ORDER EFFECTS}

\section{A. Introduction}

For circular orbits, Komorowski et al. [42] found that the numerical estimate of $(\partial \iota / \partial \tilde{l})_{\min }$ in the weak-field regime deviates from the $\partial \iota / \partial \tilde{l}$ results reported in the literature (see Flanagan and Hinderer [30], and Hughes [21]). Consider the quotient of the formulae presented in equation (3.9) of Hughes [21] where $\iota \cong \pi / 2$ :

$$
\frac{i_{\text {weak }}}{\dot{R}_{\text {weak }}}=\frac{\partial \iota}{\partial \tilde{l}}=-\frac{61}{48} \tilde{S} \tilde{l}^{-\frac{5}{2}} .
$$

Because $-61 / 48>-4.5$ in the weak-field regime, $X_{+}^{2} \Rightarrow X_{-}^{2}$ is the pertinent mode; and the best information one can obtain from $(\partial \iota / \partial \tilde{l})_{\text {min }}$ is the specification of the lower limit of $\partial \iota / \partial \tilde{l}$ for all $\tilde{l}>\tilde{l}_{L S O, \text { abutment }}$ (see [42] for more details about the abutment and its relationship to the last stable orbit (LSO)). Therefore the second-order (i.e. $\partial^{2} Q_{\text {path }} / \partial \tilde{l}^{2}$ ) behaviour at the point of tangential intersection of $Q_{X}$ and $Q_{\text {path }}$ must be considered. In section II the numerical results have been verified by analytical derivation of the formula for $(\partial \iota / \partial \tilde{l})_{\min }$ to $O\left(\tilde{S}^{3}\right)$. It remains for us to extend this analysis to include second-order effects on elliptical orbits; to this end, we shall discuss how to incorporate second-order effects into $Q_{X}$, and the resultant change to the formula for $X_{ \pm}^{2}$ (see equation (2)). Equation (20) is sufficient in treating $X_{ \pm}^{2}$, and then ultimately $\iota$, to the leading order in $\tilde{S}$. 


\section{B. Second-order Effects in $Q_{\text {path }}$}

\section{Circular orbits}

Let us begin our treatment in the $Q-\tilde{l}$ plane with the value of $e$ held constant at zero. The form of $Q_{X}$ is represented by the series in equation (10); and because $Q_{p a t h}$ intersects $Q_{X}$ tangentially at a single point $\left(\tilde{l}_{o}\right)$ (contact of the first order), we surmise:

$$
\left.Q_{p a t h}\right|_{\tilde{l}=\tilde{l}_{o}}=\left.Q_{X}\right|_{\tilde{l}=\tilde{l}_{o}}
$$

and

$$
\left.\frac{\partial Q_{p a t h}}{\partial \tilde{l}}\right|_{\tilde{l}=\tilde{l}_{o}}=\left.\frac{\partial Q_{X}}{\partial \tilde{l}}\right|_{\tilde{l}=\tilde{l}_{o}} .
$$

But the abutment can only offer an upper bound on the second derivative of $Q_{\text {path }}$, i.e.

$$
\left.\frac{\partial^{2} Q_{p a t h}}{\partial \tilde{l}^{2}}\right|_{\tilde{l}=\tilde{l}_{o}} \leqq\left.\frac{\partial^{2} Q_{X}}{\partial \tilde{l}^{2}}\right|_{\tilde{l}=\tilde{l}_{o}} .
$$

To perform an analytical treatment of the second derivative of $Q_{p a t h}$, we define an ansatz:

$$
Q_{\text {path }}=Q_{X}-\frac{\lambda^{2}}{2} f\left(\tilde{l}_{o}\right)
$$

where

$$
\begin{gathered}
f\left(\tilde{l}_{o}\right)=\left(\tilde{l}_{o}\right)^{p}(\tilde{S})^{q}\left(\sum_{k=0}^{n} a_{k}\left(\tilde{l}_{o}\right)^{-k}\right), \\
a_{0}>0
\end{gathered}
$$

and

$$
\lambda=\tilde{l}-\tilde{l}_{o}
$$

where $p$ and $q$ shall be determined by requiring that the weak-field solution be of the form, $\tilde{S} \tilde{l}^{-\frac{5}{2}}$ (see equations 27 and $(33)$ ). The adjustment represented by equation 37 is based on the Taylor expansion of a function; the function $f\left(\tilde{l}_{o}\right)$ represents a second derivative of a primitive, $\wp(e, \tilde{l})$, with respect to $\tilde{l}$, which is evaluated at $\tilde{l}_{o}$. One must not confuse the concepts of the abutment and Taylor series; equation (37) is not intended to be a Taylor series representation of $Q_{p a t h}$. We have taken the analytical formula for $Q_{X}$ and incorporated a term, which is designed to adjust the second derivative of $Q_{\text {path }}$ so that it makes contact with $Q_{X}$ tangentially at a prescribed point, $\tilde{l}_{o}$. If $\tilde{l}=\tilde{l}_{o}$, then the adjustment to $Q_{\text {path }}$ and 
$\partial Q_{\text {path }} / \partial \tilde{l}$ is zero; and the value of $\partial^{2} Q_{\text {path }} / \partial \tilde{l}^{2}$ is reduced by $f\left(\tilde{l}_{o}\right)$. Equation 37 can be applied to the analytical development of $\partial \iota / \partial \tilde{l}$. We shall call this reduction of the second derivative the reductive ansatz circular.

Let us consider how the reductive ansatz circular affects equation (2), with attention given to equations (7) and (8); although we begin with an analysis of circular orbits, $e$ has been retained in these equations for later use. Evaluate

$$
\begin{aligned}
Z_{9}\left(Q_{\text {path }}\right) & =Z_{9}\left(Q_{X}-\Phi\right) \\
& =\left[\tilde{l}^{5}+\tilde{S}^{2}\left(1-e^{2}\right)^{2} Q_{X}^{2}-\left(\tilde{l}-3-e^{2}\right) \tilde{l}^{3} Q_{X}\right] \\
& +\Phi\left(2 \tilde{S}^{2}\left(1-e^{2}\right)^{2} Q_{X}+\Phi \tilde{S}^{2}\left(1-e^{2}\right)^{2}+\left(\tilde{l}-3-e^{2}\right) \tilde{l}^{3}\right)
\end{aligned}
$$

where

$$
\Phi=\frac{\lambda^{2}}{2} f\left(\tilde{l}_{o}\right),
$$

for which the quantity in square brackets in equation 40 is equal to zero (viz. equation (8)) for all values of $\tilde{l}>\tilde{l}_{L S O \text {, abutment }}$; therefore, the use of this reductive ansatz has assured us of an effective means to simplify the expressions. The terms that remain share a common factor, $\lambda^{2}$, which will appear as $\pm \lambda$ when taken outside of the square root in equation (2). We shall limit our analysis to $O(\tilde{S})$ (the $\tilde{S}^{2}$ terms will affect terms of higher order in $\tilde{l}^{-1}$ in the series in equations (27) and (28)); therefore, the product of $Z_{7}$ (equation (5)), $Z_{8}$ (equation (6)), and $Z_{9}$ (equation(40) simplifies to:

$$
\begin{aligned}
Z_{\sqrt{\bullet}} & =Z_{7} Z_{8} Z_{9} \\
& =\Phi \tilde{l}^{5}(\tilde{l}-2(1+e))(\tilde{l}-2(1-e))\left(\tilde{l}-3-e^{2}\right),
\end{aligned}
$$

where we evaluate $Z_{\sqrt{\bullet}}$ at the point of intersection on the abutment by setting $\tilde{l}_{o}=\tilde{l}$ (i.e. $\lambda=0)$. We take the square root of $Z_{\sqrt{\bullet}}$, and a term, $\pm \lambda \sqrt{2} / 2$, emerges. The choice of sign is determined by the mode at the abutment.

Mathematically speaking there are two modes at the abutment: the fast mode

$$
X_{-}^{2} \Rightarrow X_{+}^{2}
$$

and the slow mode

$$
X_{+}^{2} \Rightarrow X_{-}^{2}
$$


In section 5 of [42] it was established that orbits that evolve on a path towards the abutment (during which $\tilde{l}>\tilde{l}_{o}$ and $\lambda>0$ ) are governed by $X_{+}^{2}$ (see equation (2)) and after making contact with the abutment at $\tilde{l}=\tilde{l}_{o}$ the orbits are then governed by $X_{-}^{2}$ (for which $\tilde{l}<\tilde{l}_{o}$ and $\lambda<0$ ) (see figure 1). Thus by choosing the positive sign for $\pm \lambda$ the equation remains consistent with the dominance of the slow mode. If one were to perform an analysis for the fast mode then $-\lambda$ would be used instead.

An examination of equations (3), (4), and (10) reveals that

$$
Z_{5}+Z_{6} Q_{X}=O\left(\tilde{S}^{2}, \tilde{l}^{4}\right)
$$

from which one may infer

$$
\begin{aligned}
& 2 \tilde{S} \sqrt{Z_{\sqrt{\bullet}}}=O\left(\tilde{S}^{2}\right) \\
& \sqrt{2} \lambda \tilde{S} \times \tilde{S}^{q / 2}=O\left(\tilde{S}^{2}\right) ;
\end{aligned}
$$

therefore, $q=2$ in the reductive ansatz (see equation (38)). The value of $p$ can be derived by considering the order of $\tilde{L}_{z}$ in $\tilde{l}$. We find (viz. equation 17 ) that $\tilde{L}_{z}=O\left(\tilde{l}^{-1}\right)$, which must not be changed by the reductive ansätze. And the leading term, $\tilde{S}$, in the expression for $X$ (see equation (12) ) must remain. Given the order of $\tilde{l}$ in equation (44), one must work with the next lower order, i.e.,

$$
\begin{aligned}
& 2 \tilde{S} \sqrt{Z_{\sqrt{\bullet}}}=O\left(\tilde{l}^{3}\right) \\
\pm & \frac{\sqrt{2}}{2} \lambda \tilde{l}^{p / 2} \tilde{l}^{4}=O\left(\tilde{l}^{3}\right) .
\end{aligned}
$$

Given $\lambda=O(\tilde{l})$, we conclude that $p=-4$. In our reductive ansätze, we have found the values of $p$ and $q$ that ensure the second-order effect does not change the form of $\partial \iota / \partial \tilde{l}$ in the weak-field regime.

\section{Elliptical orbits}

The general formulation of the reductive ansatz elliptical can be derived by starting with a Taylor series for two variables (see Appendix A 1). Because we concern ourselves with second-order effects, we shall use the following operator:

$$
\frac{1}{2 !}\left(\lambda \frac{\partial}{\partial \tilde{l}}+\epsilon \frac{\partial}{\partial e}\right)^{2}
$$


where $\lambda=\left(\tilde{l}-\tilde{l}_{o}\right)$ and $\epsilon=\left(e-e_{o}\right)$, and where the ordered pair $\left(e_{o}, \tilde{l}_{o}\right)$ specifies the location of the contact of first order between $Q_{\text {path }}$ and $Q_{X}$ (see figure 1).

One may define the reductive ansatz elliptical, i.e.

$$
\begin{aligned}
Q_{\text {path }} & =Q_{X}-\frac{1}{2}\left[\left(\lambda \frac{\partial}{\partial \tilde{l}}+\epsilon \frac{\partial}{\partial e}\right)^{2} \wp(e, \tilde{l})\right]_{e=e_{o}, \tilde{l}=\tilde{l}_{o}} \\
& =Q_{X}-\frac{\lambda^{2}}{2}\left[\frac{\partial^{2}}{\partial \tilde{l}^{2}} \wp(e, \tilde{l})\right]_{e=e_{o}, \tilde{l}=\tilde{l}_{o}}-\lambda \epsilon\left[\frac{\partial^{2}}{\partial \tilde{l} \partial e} \wp(e, \tilde{l})\right]_{e=e_{o}, \tilde{l}=\tilde{l}_{o}}-\frac{\epsilon^{2}}{2}\left[\frac{\partial^{2}}{\partial e^{2}} \wp(e, \tilde{l})\right]_{e=e_{o}, \tilde{l}=\tilde{l}_{o}}
\end{aligned}
$$

where we conjecture the existence of a primitive function, $\wp(e, \tilde{l})$. Equation 36 will also be applied to the case of elliptical orbits where $e$ is close to zero.

The expression for $Q_{\text {path }}$ is best regarded as a parameterized curve, and to make such a treatment in equation (50), one may factor out the $\lambda$, to obtain

$$
\begin{aligned}
Q_{\text {path }} & =Q_{X}-\frac{\lambda^{2}}{2}\left\{\left[\frac{\partial^{2}}{\partial \tilde{l}^{2}} \wp(e, \tilde{l})\right]_{e=e_{o} \tilde{l}=\tilde{l}_{o}}+2\left(\frac{\epsilon}{\lambda}\right)\left[\frac{\partial^{2}}{\partial \tilde{l} \partial e} \wp(e, \tilde{l})\right]_{e=e_{o}, \tilde{l}=\tilde{l}_{o}}+\left(\frac{\epsilon}{\lambda}\right)^{2}\left[\frac{\partial^{2}}{\partial e^{2}} \wp(e, \tilde{l})\right]_{e=e_{o}, \tilde{l}=\tilde{l}_{o}}\right\} \\
& =Q_{X}-\frac{\lambda^{2}}{2} g\left(e_{o}, \tilde{l}_{o}\right)
\end{aligned}
$$

for which we have the benefit of knowing the limiting form of $\epsilon / \lambda(=d e / d \tilde{l})$ to arbitrary order in $\tilde{l}^{-1}$. Thus it is possible to parameterize $Q_{\text {path }}$ in terms of $\lambda$. We can use the expression,

$$
g\left(e_{o}, \tilde{l}_{o}\right)=\tilde{S}^{2} \sum_{i=0}^{n} \frac{a_{i}\left(e_{o}\right)}{\tilde{l}_{o}^{i+4}}
$$

and this will form the basis of the reductive ansatz elliptical.

\section{Application of the Reductive Ansätze to the Analytical Derivation of $\partial \iota / \partial \tilde{l}$ and} $\partial \iota / \partial e$

The reductive ansätze (equations (37,39) and (equation (51)) constitute a reduction of the second derivative of $Q_{X}$ to more realistically model the behaviour of $Q_{\text {path }}$ at the abutment and perform a methodical treatment of this reduction in the analytical calculation of $\partial \iota / \partial \tilde{l}$ and $\partial \iota / \partial e$.

The procedure outlined in Appendix A 2 yields the following formula for $\partial \iota / \partial \tilde{l}$ :

$$
\frac{\partial \iota}{\partial \tilde{l}}=\frac{\partial[\iota(e, \tilde{l}, \lambda, \tilde{S})]_{\lambda=0}}{\partial \tilde{l}}+\left[\frac{\partial[\iota(e, \tilde{l}, \lambda, \tilde{S})]}{\partial \lambda} \frac{\partial \lambda}{\partial \tilde{l}}\right]_{\lambda=0}
$$


where

$$
\frac{\partial \lambda}{\partial \tilde{l}}=1 \text { and }\left.\lambda\right|_{\tilde{l}=\tilde{l}_{o}}=0
$$

but the result for $\partial \iota / \partial e$ is simpler,

$$
\begin{aligned}
\frac{\partial \iota}{\partial e} & =\frac{\partial[\iota(e, \tilde{l}, \lambda, \tilde{S})]_{\lambda=0}}{\partial e} \\
& =\left(\frac{\partial \iota}{\partial e}\right)_{\min } \cdot
\end{aligned}
$$

Equation (54) and the first term in equation (53) yield the formulae that describe the evolution of $\iota$ for a $Q_{\text {path }}$ along the abutment (i.e. $(\partial \iota / \partial e)_{\min }$ and $\left.(\partial \iota / \partial \tilde{l})_{\min }\right)$. The second term of equation 53 incorporates second-order effects, and thus describes the physically more realistic situation in which $Q_{\text {path }}$ intersects the abutment tangentially at a single point. Because one takes the first derivative with respect to $\lambda$, the second and higher powers of $\lambda$ will vanish when setting $\lambda=0$. But as we shall presently see, the second-order effects of the reductive ansätze remain.

We choose to work with the symbols $e$ and $\tilde{l}$ rather than $e_{o}$ and $\tilde{l}_{o}$, given that $e$ and $\tilde{l}$ can be used to represent an arbitrary point on the abutment (see Appendix A 2). The reductive ansatz circular (see equations (37,39) ) is applied at the abutment with $p=-4$, $q=2$, and $n=2$ (while retaining the two terms of leading order in $\tilde{S}$ at the conclusion of the calculation) with

$$
\frac{\partial \iota}{\partial \tilde{l}}=-\tilde{S}\left\{\frac{15}{2}+\frac{3}{2} A_{0}(0)-\frac{1}{4} A_{1}(0)\right\} \tilde{l}^{-7 / 2}-\tilde{S}\left\{\frac{9}{2}-A_{0}(0)\right\} \tilde{l}^{-5 / 2}
$$

where

$$
A_{0}(e)=+\frac{\sqrt{2}}{2} \sqrt{a_{0}(e)}, \text { and } A_{1}(e)=\frac{a_{1}(e)}{A_{0}(e)} .
$$

To apply this method to elliptical orbits, we will be required to use $g(e, \tilde{l})$. To calculate that function, the common primitive $\wp(e, \tilde{l})$ is needed.

\section{Analytical Derivation of the Common Primitive, $\wp(e, \tilde{l})$}

Now that the values of the parameters, $p=-4$ and $q=2$, have been found, it is possible to derive the formula for $\wp(e, \tilde{l})$. Consider the reductive ansatz circular:

$$
f(\tilde{l})=\tilde{S}^{2} \sum_{i=0}^{n} \frac{a_{i}}{\tilde{l}^{i+4}}
$$


We conjecture a more general form of $f(\tilde{l})$ that includes $e$ :

$$
f(e, \tilde{l})=\tilde{S}^{2} \sum_{i=0}^{n} \frac{a_{i}\left(1+b_{i} e^{2}\right)}{\tilde{l}^{i+4}} .
$$

Performing the first integration over $\tilde{l}$ yields:

$$
\begin{aligned}
F(e, \tilde{l}) & =\int f(e, \tilde{l}) d \tilde{l} \\
& =-\tilde{S}^{2}\left[\sum_{i=0}^{n}\left(\frac{1}{i+3} \frac{a_{i}\left(1+b_{i} e^{2}\right)}{\tilde{l}^{i+3}}\right)-\varkappa(e)\right] .
\end{aligned}
$$

The second integration over $\tilde{l}$ yields an expression for the common primitive:

$$
\begin{aligned}
\wp(e, \tilde{l}) & =\int F(e, \tilde{l}) d \tilde{l} \\
& =\tilde{S}^{2}\left[\sum_{i=0}^{n}\left(\frac{1}{(i+2)(i+3)} \frac{a_{i}\left(1+b_{i} e^{2}\right)}{\tilde{l}^{i+2}}\right)+\varkappa(e) \tilde{l}+\zeta(e)\right] .
\end{aligned}
$$

The constants of integration, $\varkappa(e) \tilde{l}$ and $\zeta(e)$, can each be set to zero since we require $\lim _{\tilde{l} \rightarrow \infty} \wp(e, \tilde{l})=0$. Now that the formula for $\wp(e, \tilde{l})$ is known, it is possible to obtain $g(e, \tilde{l})$, which is required by the reductive ansatz elliptical.

\section{THE TREATMENT OF $d Q / d t$ AND $d \iota / d t$ ON THE ABUTMENT}

\section{A. The $d Q / d t$ Evolution Equations}

Komorowski et al. [42] investigated the consistency of $d Q / d t$ with the evolution equation $d \tilde{l} / d t$, for circular orbits at the abutment $(\iota \gtrsim \pi / 2)$ by performing a preliminary numerical analysis for values of $\tilde{l}=\{7.0,100.0\}$ and $\mathrm{KBH}$ spin $\tilde{S}=\{0.05,0.95\}$ (see section 5.2 .1 of [42]). The published values of $d \tilde{l} / d t$ [21], which we used in our investigation, were calculated for $\iota \simeq \pi / 3$, and the difference of this value of $\iota$ from that at the abutment contributed to some inaccuracy in the analysis [42]. In this work, the derivation of analytical formulae for $\iota$ and its derivatives, as well as the use of the directional derivative to determine $d Q / d t$, now allow one to perform a more complete treatment for elliptical orbits.

Let us consider the directional derivative in equation (29) as a means of deriving $d Q / d t$ at the abutment. We have demonstrated that the second-order effects are not seen when calculating the first derivatives of $Q_{\text {path }}$ (i.e. $\partial Q_{\text {path }} / \partial \tilde{l}$ and $\partial Q_{\text {path }} / \partial e$, see equation 35 or 
(51)); therefore, we may use $\partial Q_{X} / \partial \tilde{l}$ (equation (A10) and $\partial Q_{X} / \partial e$ (equation A11) when working with equation 29 .

The form of $d Q / d t$ (equation (A.3) in [28] (after equation (56) in [27])), which was used in [42] to test $d Q / d t$ will be revisited in this work:

$$
\begin{aligned}
\left(\frac{d Q}{d t}\right)_{2 P N} & =-\left(1-\frac{1}{2} \frac{\tilde{S}^{2}\left(3+e^{2}\right)^{2}}{\tilde{l}^{3}}\right) \frac{64}{5} \frac{m^{2}}{M}\left(1-e^{2}\right)^{3 / 2} \frac{\sqrt{Q}}{\tilde{l}^{7 / 2}} \\
& \times\left[g_{9}-\frac{g_{11}}{\tilde{l}^{1}}+\pi \frac{g_{12}}{\tilde{l}^{3 / 2}}-\frac{\left(g_{13}-\tilde{S}^{2}\left(g_{14}-\frac{45}{8}\right)\right)}{\tilde{l}^{2}}\right. \\
& \left.+\tilde{S}^{2} \frac{g_{10}^{b}\left(3+e^{2}\right)}{\tilde{l}^{3}}+\frac{45}{8} \frac{\tilde{S}^{4}\left(3+e^{2}\right)^{2}}{\tilde{l}^{5}}\right] .
\end{aligned}
$$

But it is preferable that the formula for $d Q / d t$ (and for $d \iota / d t$ ) that we test be accompanied, in the same work, by their associated expressions for $d \tilde{l} / d t$ and $d e / d t$; and fortunately a paper by Ganz et al. 29] provides such information, which we shall use in our analysis. In particular, we will use equation (4.3) in [29]: the evolution equation for $\tilde{l}$,

$$
\begin{aligned}
\frac{d \tilde{l}}{d t} & =-\frac{64}{5}\left(\frac{m}{M^{2}}\right) \tilde{l}^{-3}\left(1-e^{2}\right)^{\frac{3}{2}} \\
& \times\left[g_{9}-\frac{f_{1}}{\tilde{l}}+\pi \frac{g_{12}}{\tilde{l}^{3 / 2}}+\frac{f_{3}-f_{4} \tilde{S}^{2}}{\tilde{l}^{2}}-\pi \frac{f_{7}}{\tilde{l}^{5 / 2}}\right. \\
& \left.+\frac{f_{2}\left(3+e^{2}\right) \tilde{S}^{2}}{\tilde{l}^{3}}-\frac{f_{6}\left(3+e^{2}\right) \tilde{S}^{2}}{\tilde{l}^{4}}+\frac{f_{5}\left(3+e^{2}\right)^{2} \tilde{S}^{4}}{\tilde{l}^{5}}\right],
\end{aligned}
$$

which is (excluding the common factor, $\left.\tilde{l}^{-3}\right)$ to $O\left(\tilde{l}^{-5 / 2}\right)$ in [29]; and the evolution equation for $e$,

$$
\begin{aligned}
\frac{d e}{d t} & =-\frac{304}{15}\left(\frac{m}{M^{2}}\right) \tilde{l}^{-4}\left(1-e^{2}\right)^{\frac{3}{2}} \\
& \times\left[h_{1}-\frac{h_{2}}{\tilde{l}}+\pi \frac{h_{4}}{\tilde{l}^{3 / 2}}-\frac{h_{5}+h_{6} \tilde{S}^{2}}{\tilde{l}^{2}}-\pi \frac{h_{9}}{\tilde{l}^{5 / 2}}\right. \\
& \left.+\frac{h_{3}\left(3+e^{2}\right) \tilde{S}^{2}}{\tilde{l}^{3}}-\frac{h_{8}\left(3+e^{2}\right) \tilde{S}^{2}}{\tilde{l}^{4}}+\frac{h_{7}\left(3+e^{2}\right)^{2} \tilde{S}^{4}}{\tilde{l}^{5}}\right],
\end{aligned}
$$

which is (excluding the common factor, $\left.\tilde{l}^{-4}\right)$ also to $O\left(\tilde{l}^{-5 / 2}\right)$ in [29]. 
The evolution equation of $Q$,

$$
\begin{aligned}
\left(\frac{d Q}{d t}\right)_{2.5 P N} & =-\frac{64}{5}\left(\frac{m}{M^{2}}\right) \tilde{l}^{-3}\left(1-e^{2}\right)^{\frac{3}{2}}\left(1-\frac{\tilde{S}^{2}\left(3+e^{2}\right)^{2}}{\tilde{l}^{3}}\right) \times\left[g_{9}\right. \\
& -\frac{d_{1}}{\tilde{l}^{1}}+\pi \frac{g_{12}}{\tilde{l}^{3 / 2}}-\frac{d_{3}-d_{4} \tilde{S}^{2}}{\tilde{l}^{2}}-\pi \frac{d_{7}}{\tilde{l}^{5 / 2}} \\
& \left.+\frac{\tilde{S}^{2}\left(3+e^{2}\right) d_{2}}{\tilde{l}^{3}}-\frac{\tilde{S}^{2}\left(3+e^{2}\right) d_{6}}{\tilde{l}^{4}}+\frac{\tilde{S}^{4}\left(3+e^{2}\right)^{2} d_{5}}{\tilde{l}^{5}}\right],
\end{aligned}
$$

which corresponds to equation (4.1) in [29], was of $O\left(\tilde{l}^{-5 / 2}\right)$ (excluding the common factor, $\tilde{l}^{-3}$ ). These formulae (equations (61), 62, and 63) have been converted from the variables used in 29] to our variables (see Appendices A4 and A5). Because some of the original coefficients contained $\cos (\iota)$, which we have replaced with the approximation based on equation 21, there are new terms, which correspond to $\tilde{l}^{-3}, \tilde{l}^{-4}$, and $\tilde{l}^{-5}$, in each of equations (61), 62), and (63). The original expressions did not include terms with these powers of $\tilde{l}$, so we cannot use the new terms to extend the accuracy of our analysis beyond that of the original expressions in Ganz et al. [29]. Further, these evolution equations are $O\left(e^{2}\right)$, hence the final results must also be used up to the second power of $e$.

We assume that the evolution of the orbit, $d \tilde{l} / d t$ and $d e / d t$, is described by equations 61 . and (62) (from Ganz et al. [29]); the result of evaluating equation (29) is compiled in table [1 (first column). The second column contains the result $(d Q / d t)_{2.5 P N}$, derived by Ganz et al. (see equation (63)), evaluated on the abutment. Similarly, the third column contains the formula for $(d Q / d t)_{2 P N}$ based on equation (A.3) in [28], also evaluated on the abutment.

Although the terms in table $\mathrm{I}$ are $O\left(e^{4}\right)$ (N.B. Those given to $O\left(e^{2}\right)$ were found to have no terms of higher order in $e^{2}$ ), any final result one might calculate must be of $O\left(e^{2}\right)$. To that order, our calculated results at the abutment agree with those of Ganz et al. [29] up to $\tilde{l}^{-5 / 2}$. There is also agreement with Barausse, Hughes, and Rezzolla [28] up to $\tilde{l}^{-2}$ with the exception of the coefficient for the $\tilde{S}^{2}$ term in the third column (marked with $\S$ ), which differs from the other two results; the expanded equation in Barausse, Hughes, and Rezzolla differed from that of Ganz for that order of $\tilde{l}$.

There are two reasons for reporting these results to $O\left(e^{4}\right)$ : first, we wish to demonstrate that the confirmation of Ganz's calculations is not to be dismissed as a fortuitous triviality. The method of calculation of $d Q / d t$ at the abutment differs fundamentally from that used by Ganz et al. to derive their results, as would be required of a good consistency condition; 
Table I: The coefficients of $-5 / 64 M^{2} / m \tilde{l}^{3}\left(1-e^{2}\right)^{-3 / 2} d Q / d t$. The first column contains our calculated results at the abutment. The second column contains the results of Ganz et al. [29], and the third column contains the results of Barausse, Hughes, and Rezzolla [28]. The trigonometric functions in both of these sets were evaluated on the abutment. Note: although the terms are reported to $O\left(e^{4}\right)$ they are only accurate to $O\left(e^{2}\right)$.

\begin{tabular}{|c|c|c|c|}
\hline & $\begin{array}{l}\text { Results at the Abutment } \\
\text { (see equation }(29) \text { ) }\end{array}$ & $\begin{array}{l}\text { (equation } 4.1 \text { in [29]) } \\
\text { (see equation } 63 p)\end{array}$ & $\begin{array}{l}\text { (equation }(\mathrm{A} 3) \text { in }[28] \text { ) } \\
\text { (see equation } 600)\end{array}$ \\
\hline & $1+\frac{7}{8} e^{2}$ & $1+\frac{7}{8} e^{2}$ & $1+\frac{7}{8} e^{2}$ \\
\hline$\tilde{l}^{-1}$ & $-\left(\frac{743}{336}-\frac{23}{42} e^{2}-\frac{121}{96} e^{4}\right)$ & $-\left(\frac{743}{336}-\frac{23}{42} e^{2}\right)$ & $-\left(\frac{743}{336}-\frac{23}{42} e^{2}-\frac{7}{16} e^{4}\right)$ \\
\hline$\tilde{l}^{-3 / 2}$ & $\pi\left(4+\frac{97}{8} e^{2}\right)$ & $\pi\left(4+\frac{97}{8} e^{2}\right)$ & $\pi\left(4+\frac{97}{8} e^{2}\right)$ \\
\hline$\tilde{l}^{-2}$ & $-\left[\frac{129293}{18144}+\frac{84035}{1728} e^{2}-\frac{629}{672} e^{4}\right.$ & $-\left[\frac{129293}{18144}+\frac{84035}{1728} e^{2}\right.$ & $-\left[\frac{129293}{18144}+\frac{84035}{1728} e^{2}-\frac{575}{336} e^{4}\right.$ \\
\hline & $\left.+\tilde{S}^{2}\left(\frac{329}{96}+\frac{929}{96} e^{2}\right)\right]$ & $\left.+\tilde{S}^{2}\left(\frac{329}{96}+\frac{929}{96} e^{2}\right)\right]$ & $\left.+\tilde{S}^{2}\left(\frac{342}{96}-\frac{570}{96} e^{2}\right)\right] \S$ \\
\hline$\tilde{l}^{-5 / 2}$ & $-\pi\left(\frac{4159}{672}+\frac{21229}{1344} e^{2}-\frac{41783}{1344} e^{4}\right)$ & $-\pi\left(\frac{4159}{672}+\frac{21229}{1344} e^{2}\right)$ & $\pi\left(\frac{4032}{672}+\frac{27132}{1344} e^{2}+\frac{8148}{1344} e^{4}\right)$ \\
\hline \multirow[t]{2}{*}{$\tilde{l}^{-3}$} & $-\left[\frac{3819}{112}+\frac{770993}{12096} e^{2}+\frac{333937}{2304} e^{4}\right.$ & $-[0$ & - \\
\hline & $\left.-\tilde{S}^{2}\left(\frac{250}{8}+\frac{7261}{192} e^{2}-\frac{821}{384} e^{4}\right)\right]$ & $\left.-\tilde{S}^{2}\left(\frac{183}{8}+\frac{607}{8} e^{2}+\frac{161}{8} e^{4}\right)\right]$ & - \\
\hline
\end{tabular}

second, the differing coefficient values for the $e^{4}$ terms demonstrate that one must not perform calculations on the abutment for highly eccentric orbits. While the abutment equations $\left(\partial Q_{X} / \partial \tilde{l}, \partial Q_{X} / \partial e\right.$, and $\left.\iota(\tilde{l}, e, \tilde{S})\right)$ are exact in terms of $e^{2}$, one remains limited by the order of $e$ used in the radiation back-reaction model being tested.

Because the expressions for $\partial Q_{X} / \partial \tilde{l}$ and $\partial Q_{X} / \partial e$ can be derived to arbitrary order in $\tilde{l}^{-1}$, and the coefficients for each power are exact finite series in $e^{2}$, it is worthwhile to consider using the abutment to improve the order of $e^{2}$ of the evolution equations in the weaker field regime by allowing other theoreticians to perform a test of their own, improved backreaction models. Since the abutment extends down to the LSO, one might also explore the development and testing of evolution equations in the strong-field regime, given that on the abutment the trigonometric contributions of $\sin (\iota)$ and $\cos (\iota)$ can be expressed as functions of $e, \tilde{l}$, and $\tilde{S}$. But one must also be mindful of the assumptions made at the outset of this exercise, in particular, the assumption that the secondary object can be approximated as a test-particle of infinitesimal mass, and the use of adiabatically evolving orbits. 


\section{B. The Second-order Calculation of $d \iota / d t$ for the Leading Order of $\tilde{S}$ (weak-field} regime)

Now that $\wp(e, \tilde{l})$ is known we can calculate $g(e, \tilde{l})$; but let us first derive $d e / d \tilde{l}$ using equations 61 and 62 . We find the following:

$$
\frac{d e}{d \tilde{l}}=\frac{19}{12} e\left(\frac{1-\frac{145}{304} e^{2}}{\tilde{l}}+\frac{\frac{3215}{3192}-\frac{33373}{102144} e^{2}}{\tilde{l}^{2}}\right),
$$

expressed to $O\left(\tilde{l}^{-2}\right)$. From equation 51 , one derives:

$$
g(e, \tilde{l})=\tilde{S}^{2}\left[\frac{a_{0}(e)}{\tilde{l}^{4}}+\frac{a_{1}(e)}{\tilde{l}^{5}}\right]
$$

where

$$
a_{0}(e)=a_{0}(0)\left(1-\frac{119}{432} b_{0} e^{2}\right)
$$

and

$$
a_{1}(e)=a_{1}(0)\left(1+\left(\frac{3215}{72576} b_{0}-\frac{143}{864} b_{1}\right) e^{2}\right),
$$

which can be used to calculate $\partial \iota / \partial \tilde{l}$ under the reductive ansatz elliptical,

$$
\begin{aligned}
\frac{\partial \iota}{\partial \tilde{l}} & =-\tilde{S}\left\{\frac{5}{2}\left(3+e^{2}\right)\left(1+e^{2}\right)+\frac{1}{2}\left(3+e^{2}\right) A_{0}(e)-\frac{1}{4} A_{1}(e)\right\} \tilde{l}^{-7 / 2} \\
& -\tilde{S}\left\{\frac{3}{2}\left(3+e^{2}\right)-A_{0}(e)\right\} \tilde{l}^{-5 / 2}
\end{aligned}
$$

where

$$
A_{0}(e)=A_{0}(0)\left(1-\frac{1}{2} \frac{119}{432} b_{0} e^{2}\right)
$$

and

$$
A_{1}(e)=\frac{a_{1}(e)}{A_{0}(e)} .
$$

Now that we have developed a formula for $\partial \iota / \partial \tilde{l}$ (equation $(66)$ ) that incorporates the reductive ansatz elliptical, and we have found that $\partial \iota / \partial e$ (equation(28) is unaffected by the reductive ansatz elliptical, the expression for $d \iota / d t$ can be obtained from equation (30) to the leading order in $\tilde{S}$ with coefficients of $O\left(e^{2}\right)$ :

$$
\frac{d \iota}{d t}=\tilde{S} \frac{m}{M^{2}} \tilde{l}^{-4}\left(1-e^{2}\right)^{\frac{3}{2}}\left(\frac{U_{1}}{\tilde{l}^{3 / 2}}-\frac{U_{3}}{\tilde{l}^{5 / 2}}\right),
$$


where

$$
\begin{aligned}
U_{1} & =\frac{32}{15}\left(9-2 A_{0}(e)\right)+\frac{4}{15}\left(-109+42 A_{0}(e)\right) e^{2} \\
U_{3} & =\frac{2}{105}\left(1647-2494 A_{0}(e)+168 A_{1}(e)\right) \\
& +\frac{1}{105}\left(682-2978 A_{0}(e)+147 A_{1}(e)\right) e^{2} .
\end{aligned}
$$

Equation A15 can be expanded and expressed to leading order in $\tilde{S}$ to yield:

$$
\frac{d \iota}{d t}=\frac{244}{15} \tilde{S} \frac{m}{M^{2}} \tilde{l}^{-4}\left(1-e^{2}\right)^{\frac{3}{2}}\left(\frac{u_{1}}{\tilde{l}^{3 / 2}}-\frac{u_{3}}{\tilde{l}^{5 / 2}}\right) .
$$

By equating the terms in equations (67) and (68) (i.e. $U_{1}=244 u_{1} / 15$ and $U_{3}=244 u_{3} / 15$ ) one first solves for $A_{0}(0)$ and $A_{1}(0)$ for a circular orbit by setting $e=0$ :

$$
A_{0}(0)=\frac{155}{48}
$$

and

$$
A_{1}(0)=\frac{279289}{4032}
$$

By substituting these values into equation (67), we obtain,

$$
\begin{aligned}
\frac{d \iota}{d t} & =\frac{244}{15} \tilde{S} \frac{m}{M^{2}} \tilde{l}^{-4}\left(1-e^{2}\right)^{\frac{3}{2}} \\
& \times\left[\frac{\left(1+\left(\frac{18445}{52704} b_{0}-\frac{213}{488}\right) e^{2}\right)}{\tilde{l}^{3 / 2}}\right. \\
& \left.-\frac{\left(\frac{10461}{1708}+\left(\frac{79869}{54656}-\frac{39938327}{17708544} b_{1}+\frac{5621763839}{1487517696} b_{0}\right) e^{2}\right)}{\tilde{l}^{5 / 2}}\right] .
\end{aligned}
$$

When evaluated at $e=0$, the expression in equation (69) matches the results reported in the literature (equations (31), (32), and (68)) for near-polar orbits. For near-circular orbits, values of $b_{0}$ and $b_{1}$ can be found for which the coefficients of the $\tilde{l}^{-3 / 2}$ and $\tilde{l}^{-5 / 2}$ terms in equation 69 match their theoretical counterparts in equation 68.

\section{The Independence of the Abutment of Radiation Back-reaction Models}

Let us clarify the meaning of our statement that the abutment model is independent of any specific radiation back-reaction model. The expression for the abutment, $Q_{X}$ (equation (9)), is determined by the characteristics of the Kerr spacetime of the primary object in 
which the secondary object (i.e. test-particle) orbits. The analytical expressions for $d \tilde{l} / d t$ and $d e / d t$ describe the effects of radiation back-reaction on the values of $\tilde{l}$ and $e$ of the orbit, and they serve as inputs to our abutment model in two ways: first, through the quotient $\epsilon / \lambda \cong \partial e / \partial \tilde{l}$ (equation (51)); and second, through the directional derivatives in equations (29) and (30).

The mechanics of the abutment remain consistent, the details of the radiation backreaction model notwithstanding. The results of either directional derivative are outputs of the abutment model that describe the effect of the radiation back-reaction on the listing of the test-particle orbit.

\section{CONCLUSIONS}

For inclined test-particle orbits around a black hole, two solutions for $X^{2}$ (where $X=$ $\left.\tilde{L}_{z}-\tilde{S} \tilde{E}\right)$ can be derived: $X_{-}^{2}$ and $X_{+}^{2}$. Given a Schwarzschild black hole (SBH), $X_{+}^{2}=X_{-}^{2}$ on any polar orbit, where $X_{-}^{2}$ corresponds to prograde orbits and $X_{+}^{2}$ corresponds to retrograde orbits. For a Kerr black hole $(\mathrm{KBH})$ the orbits on which $X_{+}^{2}=X_{-}^{2}$ are not polar, but near-polar and retrograde. Such orbits comprise the abutment at which the value of the Carter constant $(Q)$ is a maximum for given values of latus rectum $(\tilde{l})$ and eccentricity $(e)$.

In this work we derived an analytical formula for the value of orbital inclination, $\iota$, of an elliptical orbit on the abutment. By performing the partial differentiation of $\iota$ with respect to $\tilde{l}$, we were able to confirm the numerical result for $\partial \iota / \partial \tilde{l}$ reported in Komorowski et al. [42] for circular orbits, and we were able to extend the formula to include $\partial \iota / \partial \tilde{l}$ for elliptical orbits. A result for $\partial \iota / \partial e$ was also obtained for elliptical orbits. Further, it allowed one to redefine, in terms of $e, \tilde{l}$, and $\tilde{S}$, any trigonometric function that might be found in an evolution equation to be tested at the abutment.

Evolving orbits in Kerr spacetime are not constrained to follow the abutment. Instead, the value of $Q$ will follow $Q_{\text {path }}$, which intersects the abutment tangentially at an arbitrary point of contact of the first order. This behaviour is assured because the value of $Q_{\text {path }}$ cannot exceed that of $Q$ on the abutment; to do so would make $X_{ \pm}^{2}$ complex and thus unphysical. For circular orbits, we modelled the second-order behaviour reported in 42 by introducing a bounded function $f(e, \tilde{l})$ (also in terms of $\tilde{S}$ ) to reduce the value of $\partial^{2} Q_{p a t h} / \partial \tilde{l}^{2}$ while leaving $Q_{\text {path }}$ and $\partial Q_{\text {path }} / \partial \tilde{l}$ equal to their corresponding values $\left(Q_{X}\right.$ and $\left.\partial Q_{X} / \partial \tilde{l}\right)$ on the 
abutment. This approach was then applied to elliptical orbits, and a new bounded function

$g(e, \tilde{l})$, which depends upon $d e / d \tilde{l}$, was used to reduce the value of $\partial^{2} Q_{X} / \partial \tilde{l}^{2}$ to $\partial^{2} Q_{p a t h} / \partial \tilde{l}^{2}$. It was discovered that the value of $\partial^{2} Q_{\text {path }} / \partial e^{2}$ remained unchanged by the reductive ansatz elliptical.

The consistency of published evolution equations, $d Q / d t, d \tilde{l} / d t$, and $d e / d t$, was tested by using $d \tilde{l} / d t$ and $d e / d t$ to generate an expression for $d Q / d t$ at the abutment. In general, the calculation of $d Q / d t$ is more difficult to perform than that of $d \tilde{l} / d t$ and $d e / d t$ [19]; hence, the abutment provides a useful mechanism for testing the validity of radiation back-reaction models. Indeed, the evolution equations reported by Ganz et al. [29] were confirmed to their 2.5PN order. The abutment provides a consistency condition that is limited to nearpolar retrograde orbits; and yet, some back-reaction models have been found to exhibit pathological behaviour for polar orbits (Gair and Glampedakis [27]). Another consistency condition is already known for orbits of any $\iota$ (see equations (13) and (14) in [27]), but it applies to circular orbits (i.e. in the limit $e \rightarrow 0$ ); the abutment is valid for orbits of arbitrary eccentricity, depending on the accuracy of the back-reaction model used.

This method promises to be a useful tool for confirming the accuracy of evolution equations to greater order in $e$ and $\tilde{l}^{-1}$. Further work might entail the development of a more precise mathematical treatment of the ansätze in relation to the underlying physical concepts of the radiation back-reaction process and its effect on the listing behaviour of orbits near the abutment. It would also be intriguing to investigate the consistency condition reported by Gair and Glampedakis [27], on the abutment.

\section{Acknowledgments}

The authors are very grateful to Dr. Daniel Kennefick for his continued encouragement and for his invaluable discussions of this manuscript. MH's research is funded through the NSERC Discovery Grant, Canada Research Chair, Canada Foundation for Innovation, Ontario Innovation Trust and Western's Academic Development Fund programs. SRV would like to acknowledge the Faculty of Science for a UWO Internal Science Research Grant award during the progress of this work. 


\section{Appendix A: Ancillary Equations}

\section{Taylor Series for two Variables}

Refer to Chapter 6 in [44 for a more detailed treatment. Let us consider a locally continuous function with two independent variables, $f(x, y)$. We may use an operator

$$
\left(h \frac{\partial}{\partial x}+k \frac{\partial}{\partial y}\right)
$$

to construct a Taylor series of $n$ terms

$$
\begin{aligned}
f\left(x_{o}+h, y_{o}+k\right) & =f\left(x_{o}, y_{o}\right) \\
& +\left[\left(h \frac{\partial}{\partial x}+k \frac{\partial}{\partial y}\right) f(x, y)\right]_{x=x_{o}, y=y_{o}}+\frac{1}{2 !}\left[\left(h \frac{\partial}{\partial x}+k \frac{\partial}{\partial y}\right)^{2} f(x, y)\right]_{x=x_{o}, y=y_{o}} \\
& \ldots+\frac{1}{n !}\left[\left(h \frac{\partial}{\partial x}+k \frac{\partial}{\partial y}\right)^{n} f(x, y)\right]_{x=x_{o}, y=y_{o}}
\end{aligned}
$$

if the $(n+1)^{t h}$ partial derivatives are continuous. In this paper, we are concerned only with the second derivative.

\section{Treatment of the Taylor Series Under Partial Differentiation}

Given the term:

$$
A=h f\left(x_{o}\right) g(x),
$$

where $h=\left(x-x_{o}\right)$. We can calculate the partial derivative of $A$ with respect to $x$,

$$
\begin{aligned}
\frac{\partial A}{\partial x} & =\frac{\partial}{\partial x}\left(h f\left(x_{o}\right) g(x)\right) \\
& =f\left(x_{o}\right) \frac{\partial}{\partial x}(h g(x)) \\
& =f\left(x_{o}\right)\left(h \frac{\partial}{\partial x} g(x)+g(x) \frac{\partial}{\partial x} h\right),
\end{aligned}
$$

and thus demonstrate

$$
\left.\frac{\partial A}{\partial x}\right|_{x=x_{o}}=f\left(x_{o}\right) g\left(x_{o}\right) .
$$


Consider a more complicated case where we have a function $F(x, h, y, k)$, where $h=$ $\left(x-x_{o}\right)$ and $k=\left(y-y_{o}\right)$. Calculate

$$
\left.\frac{\partial F}{\partial x}\right|_{x=x_{o}} \text { and }\left.\frac{\partial F}{\partial y}\right|_{y=y_{o}} .
$$

If we hold $y$ constant and set $k=0$, then

$$
\frac{d F}{d x}=\frac{\partial F}{\partial x}+\frac{\partial F}{\partial h} \frac{\partial h}{\partial x}
$$

If we hold $x$ constant and set $h=0$, then

$$
\frac{d F}{d y}=\frac{\partial F}{\partial y}+\frac{\partial F}{\partial k} \frac{\partial k}{\partial y}
$$

These results will be of use in applying the second-order effects to $Q_{p a t h}$ as it makes contact with the abutment, $Q_{X}$.

\section{Treatment of $Q_{X}$ as a Series in $\tilde{l}$}

The expansion of $Q_{X}$ in terms of $\tilde{S}$ (equation 10 ) can be expressed as a series in $\tilde{l}$ :

$$
Q_{X}=\tilde{l}+\sum_{i=0}^{\infty}\left[\frac{\left(3+e^{2}\right)^{i+1}}{\tilde{l}^{i}}+\frac{i(i-1)}{2} \frac{\left(3+e^{2}\right)^{i-2}\left(1-e^{2}\right)^{2}}{\tilde{l}^{i}} \tilde{S}^{2}\right] .
$$

From equation $\mathrm{A} 9 \mathrm{p}$ one can obtain $\partial Q_{X} / \partial \tilde{l}$ and $\partial Q_{X} / \partial e$ directly:

$$
\frac{\partial Q_{X}}{\partial \tilde{l}}=1-\sum_{i=0}^{\infty}\left[\frac{i\left(3+e^{2}\right)^{i+1}}{\tilde{l}^{i+1}}+\frac{i^{2}(i-1)}{2} \frac{\left(3+e^{2}\right)^{i-2}\left(1-e^{2}\right)^{2}}{\tilde{l}^{i+1}} \tilde{S}^{2}\right],
$$

and

$$
\begin{aligned}
\frac{\partial Q_{X}}{\partial e} & =2 e \sum_{i=0}^{\infty}\left[\frac{(i+1)\left(3+e^{2}\right)^{i}}{\tilde{l}^{i}}-i(i-1) \frac{\left(3+e^{2}\right)^{i-2}\left(1-e^{2}\right)}{\tilde{l}^{i}} \tilde{S}^{2}\right. \\
& \left.+\frac{i(i-1)(i-2)}{2} \frac{\left(3+e^{2}\right)^{i-3}\left(1-e^{2}\right)^{2}}{\tilde{l}^{i}} \tilde{S}^{2}\right] .
\end{aligned}
$$


The $\sqrt{Q_{X}}$ will also be required for the treatment of $d Q / d t$, (see Appendix A 4 )

$$
\begin{aligned}
\sqrt{Q_{X}} & =\sqrt{\tilde{l}}+\frac{1}{2} \frac{\left(3+e^{2}\right)}{\sqrt{\tilde{l}}}+\frac{3}{8} \frac{\left(3+e^{2}\right)^{2}}{\tilde{l}^{3 / 2}}+\frac{1}{2} \frac{\left(\left(1-e^{2}\right)^{2} \tilde{S}^{2}+\frac{5}{8}\left(3+e^{2}\right)^{3}\right)}{\tilde{l}^{5 / 2}} \\
& +\frac{5}{128} \frac{\left(3+e^{2}\right)\left(32\left(1-e^{2}\right)^{2} \tilde{S}^{2}+7\left(3+e^{2}\right)^{3}\right)}{\tilde{l}^{7 / 2}} \\
& +\frac{7}{256} \frac{\left(3+e^{2}\right)^{2}\left(80\left(1-e^{2}\right)^{2} \tilde{S}^{2}+9\left(3+e^{2}\right)^{3}\right)}{\tilde{l}^{9 / 2}}+\ldots
\end{aligned}
$$

\section{The $2 \mathrm{PN}$ Flux for $Q$}

Equation (60), an expression for $d Q / d t$, was derived from equation (A.3) in [28] (after equation (56) in [27]) by substituting the approximations of $\sin (\iota)$ and $\cos (\iota)$ on the abutment. where

$$
\begin{gathered}
g_{9}=1+\frac{7}{8} e^{2}, \quad g_{10}^{b}=\frac{61}{8}+\frac{91}{4} e^{2}+\frac{461}{64} e^{4}, \quad g_{11}=\frac{1247}{336}+\frac{425}{336} e^{2}, \\
g_{12}=4+\frac{97}{8} e^{2}, \quad g_{13}=\frac{44711}{9072}+\frac{302893}{6048} e^{2}, \quad g_{14}=\frac{33}{16}+\frac{95}{16} e^{2} .
\end{gathered}
$$

An alternative expression for $d Q / d t$ to $2.5 \mathrm{PN}$ order was presented by Ganz et al. (equation (4.1) in [29]) in which we have converted their variable, $Y \simeq \cos (\iota)$, to $-\tilde{S}\left(3+e^{2}\right) \tilde{l}^{-3 / 2}$ to yield equation (63), where

$$
\begin{gathered}
d_{1}=\frac{743}{336}-\frac{23}{42} e^{2}, \quad d_{2}=\frac{85}{8}+\frac{211}{8} e^{2} \quad d_{3}=\frac{129193}{18144}+\frac{84035}{1728} e^{2}, \\
d_{4}=\frac{329}{96}+\frac{929}{96} e^{2}, \quad d_{5}=\frac{53}{8}+\frac{163}{8} e^{2}, \quad d_{6}=\frac{2553}{224}-\frac{553}{192} e^{2}, \quad d_{7}=\frac{4159}{672}+\frac{21229}{1344} e^{2} .
\end{gathered}
$$

\section{Evolution Equations for $\tilde{l}, e$, and $\iota$}

The evolution equations for $\tilde{l}, e$, and $\iota$ are reported by Ganz et al. (see equation (4.3) in [29]) to $O\left(\tilde{l}^{-5 / 2}\right)$. We reproduce them in equations $61, \sqrt{62}$, and 415 after having converted their original variable, $v=\sqrt{\frac{M}{l}}$, to $\tilde{l}^{-1 / 2}$, and by using $d v=-1 / 2 \tilde{l}^{-3 / 2} d \tilde{l}$, where

$$
\begin{gathered}
f_{1}=\frac{743}{336}+\frac{55}{21} e^{2}, \quad f_{2}=\frac{133}{12}+\frac{379}{24} e^{2}, \quad f_{3}=\frac{34103}{18144}-\frac{526955}{12096} e^{2}, \\
f_{4}=\frac{329}{96}+\frac{929}{96} e^{2}, \quad f_{5}=\frac{815}{96}+\frac{477}{32} e^{2}, \quad f_{6}=\frac{1451}{56}+\frac{1043}{96} e^{2}
\end{gathered}
$$




$$
f_{7}=\frac{4159}{672}+\frac{48809}{1344} e^{2}
$$

and where

$$
\begin{gathered}
h_{1}=1+\frac{121}{304} e^{2}, \quad h_{2}=\frac{6849}{2128}+\frac{4509}{2128} e^{2}, \quad h_{3}=\frac{879}{76}+\frac{515}{76} e^{2}, \\
h_{4}=\frac{985}{152}+\frac{5969}{608} e^{2}, \quad h_{5}=\frac{286397}{38304}+\frac{2064415}{51072} e^{2}, \quad h_{6}=\frac{3179}{608}+\frac{8925}{1216} e^{2}, \\
h_{7}=\frac{5869}{608}+\frac{10747}{1216} e^{2}, \quad h_{8}=\frac{1903}{304}-\frac{22373}{8512} e^{2}, \quad h_{9}=\frac{87947}{4256}+\frac{4072433}{68096} e^{2} .
\end{gathered}
$$

We use the following equations to convert the form of the equation for list rate in [29]:

$$
\begin{aligned}
1- & Y^{2} \simeq \sin ^{2}(\iota) \\
\frac{d Y}{d t} & =\frac{d \cos (\iota)}{d \iota} \times \frac{d \iota}{d t} \\
& =-\sin (\iota) \times \frac{d \iota}{d t} .
\end{aligned}
$$

We obtain:

$$
\begin{aligned}
\frac{d \iota}{d t} & =\frac{244}{15}\left(\frac{m}{M^{2}}\right) \tilde{l}^{-4}\left(1-e^{2}\right)^{\frac{3}{2}}\left(1-\frac{1}{2} \tilde{S}^{2}\left(3+e^{2}\right)^{2} \tilde{l}^{-3}\right) \\
& \times\left[\frac{u_{1} \tilde{S}}{\tilde{l}^{3 / 2}}-\frac{u_{3} \tilde{S}}{\tilde{l}^{5 / 2}}+\frac{u_{2}\left(3+e^{2}\right) \tilde{S}^{3}}{\tilde{l}^{7 / 2}}\right] \\
& =\frac{244}{15}\left(\frac{m}{M^{2}}\right) \tilde{l}^{-4}\left(1-e^{2}\right)^{\frac{3}{2}} \\
& \times\left[\frac{u_{1} \tilde{S}}{\tilde{l}^{3 / 2}}-\frac{u_{3} \tilde{S}}{\tilde{l}^{5 / 2}}+\frac{u_{2}\left(3+e^{2}\right) \tilde{S}^{3}}{\tilde{l}^{7 / 2}}\right. \\
& \left.-\frac{1}{2} \frac{u_{1}\left(3+e^{2}\right)^{2} \tilde{S}^{3}}{\tilde{l}^{9 / 2}}+\frac{1}{2} \frac{u_{3}\left(3+e^{2}\right)^{2} \tilde{S}^{3}}{\tilde{l}^{11 / 2}}-\frac{1}{2} \frac{u_{2}\left(3+e^{2}\right)^{3} \tilde{S}^{5}}{\tilde{l}^{13 / 2}}\right]
\end{aligned}
$$

where

$$
u_{1}=1+\frac{189}{61} e^{2}, \quad u_{2}=\frac{13}{244}+\frac{277}{244} e^{2}, \quad u_{3}=\frac{10461}{1708}+\frac{83723}{3416} e^{2} .
$$

\section{Appendix B: Series Expansions of Critical Values in Terms of $\tilde{S}$ and $\tilde{l}$}

Our conversion of the quantities $X$ (equation $(12)$ ), $\tilde{E}$ (equation $(13)$ ), and $Q_{X}$ (equation (10) ) to expansion series in $\tilde{S}$ helped to simplify our analysis by avoiding the use of the 
much more complicated series expansions in terms of $\tilde{l}$. Equation 19 can be converted to a series:

$$
\frac{\tilde{L}_{z}^{(n+1)}}{\sqrt{Q_{X}}}=\sum_{i=0}^{n} c_{2 i+1} \tilde{S}^{2 i+1}
$$

By choosing the order of $\tilde{S}$ (the value of $2 n+1$ ) in which to work, it becomes easier to derive suitable series approximations of these quantities, and their mathematical combinations, in terms of $\tilde{l}$. Since the equations derived during the full analytical treatment are Brobdingnagian, and thus preclude detailed presentation in this paper, we shall offer the essential highlights of our analysis.

\section{First-order Calculations}

We require the series expansion of the quotient, which appears in equation (20),

$$
\frac{\stackrel{(1)}{L}_{z}}{\sqrt{Q_{X}}}
$$

to be expressed in terms of $\tilde{l}$. To obtain this result we perform a careful manipulation of $\tilde{L}_{z}$ (in terms of $X$ and $\tilde{E}$, viz. equation 17 ) and $Q_{X}$ (as a series expansion in $\tilde{S}$ ) using MacLaurin series. The coefficient of $\tilde{S}^{1}$ (i.e. $c_{1}$ ) is converted to an expansion in $\tilde{l}$; we find $c_{1}$ to be:

$$
c_{1}=\frac{\psi_{1}-\psi_{2}}{\sqrt{\frac{\tilde{l}^{2}}{\tilde{l}-e^{2}-3}}}
$$

where

$$
\begin{gathered}
\psi_{1}=\sqrt{\frac{\tilde{l}\left(\tilde{l}^{2}-4\left(\tilde{l}+e^{2}-1\right)\right)}{\left(\tilde{l}-e^{2}-3\right)^{3}}} \\
\psi_{2}=\sqrt{\frac{\tilde{l}^{2}-4\left(\tilde{l}+e^{2}-1\right)}{\tilde{l}\left(\tilde{l}-e^{2}-3\right)} .}
\end{gathered}
$$

From equation (B3), one obtains the result:

$$
c_{1}=-\left(e^{2}+3\right)\left(\frac{1}{\tilde{l}^{3 / 2}}+\frac{\left(1+e^{2}\right)}{\tilde{l}^{5 / 2}}+\frac{\left(3+2 e^{2}+e^{4}\right)}{\tilde{l}^{7 / 2}}+\frac{\left(9+5 e^{2}+5 e^{4}+e^{6}\right)}{\tilde{l}^{9 / 2}}\right),
$$

which appears in equation 21. 


\section{Third-order Calculations}

The third-order calculations require two additional factors:

$$
\frac{\tilde{L}_{z}}{\sqrt{Q_{X}}} \text { and } \frac{\tilde{S}^{2}\left(1-\tilde{E}^{2}\right)}{Q_{X}}
$$

which are used to evaluate $x=\cos (\iota)$ using equation (23). The first factor can be derived by converting the coefficient of $\tilde{S}^{3}$,

$$
c_{3}=-\frac{1}{2} \frac{\left(1-e^{2}\right)^{2}\left(\left(\tilde{l}^{2}-2 \tilde{l}+2 \tilde{l} e^{2}-16 e^{2}\right) \psi_{1}+(-4+\tilde{l})\left(\tilde{l}-2-2 e^{2}\right) \psi_{2}\right)}{\sqrt{\frac{\tilde{l}^{2}}{\tilde{l}-e^{2}-3}} \tilde{l}\left(\tilde{l}-e^{2}-3\right)\left(\tilde{l}^{2}-4 \tilde{l}+4 e^{2}-4\right)},
$$

in equation $\mathrm{B} 1 \mathrm{l}$, to a series expansion in $\tilde{l}$ (see equation 24) and adding the result to the first order term:

$$
c_{3}=-\tilde{S}^{3}\left(1-e^{2}\right)^{2}\left(\frac{1}{\tilde{l}^{7 / 2}}+\frac{1}{2} \frac{11+5 e^{2}}{\tilde{l}^{9 / 2}}\right)
$$

(see equation (24)). The second factor in equation (B5) is also obtained by working in

expansions of $\tilde{S}$, which proceeds by a simpler derivation (see equation 25 ). The orbital inclination, $\iota$, is then obtained by using equation $(22)$.

[1] J. M. Bardeen, in C. Dewitt and B. S. Dewitt (Ed.), Black Holes (Les Astres Occlus), (Gordon and Breach, New York, London, and Paris, 1973) 215.

[2] E. Poisson, Phys Rev D 47, 1497 (1993).

[3] C. Cutler, L. S. Finn, E. Poisson, and G. J. Sussman, Phys Rev D 47, 1511 (1993).

[4] F. D. Ryan, Phys Rev D 52, 3159 (1995).

[5] A. Ori and K. S. Thorne, Phys Rev D 62, 124022 (2000).

[6] D. Pugliese, H. Quevedo, and R. Ruffini, Phys Rev D 84 (2011) 044030.

[7] P. C. Peters and J. Mathews, Phys Rev 131, 435 (1963).

[8] P. C. Peters, Phys Rev 136, B1224 (1964).

[9] V. A. Brumberg, Essential Relativistic Celestial Mechanics (IOP Publishing, Bristol, UK, 1991). 
[10] W. Junker and G. Schaefer, Mon Not R Astron Soc 254, 146 (1992).

[11] C. Cutler, D. Kennefick, and E. Poisson, Phys Rev D 50, 3816 (1994).

[12] K. Glampedakis and D. Kennefick, Phys Rev D 66, 044002 (2002).

[13] S. A. Hughes, S. Drasco, .. Flanagan, and J. Franklin, Phys Rev Lett 94, 221101 (2005).

[14] S. A. Teukolsky, Astrophys J 185, 635 (1973).

[15] W. H. Press and S. A. Teukolsky, Astrophys J 185, 649 (1973).

[16] A. Ori, Phys Lett A 202, 347 (1995).

[17] F. D. Ryan, Phys Rev D 53, 3064 (1996).

[18] D. Kennefick and A. Ori, Phys Rev D 53, 4319 (1996).

[19] A. Ori, Phys Rev D 55, 3444 (1997).

[20] F. de Felice and G. Preti, Classical Quant Grav 16, 2929 (1999).

[21] S. A. Hughes, Phys Rev D 61, 084004 (2000).

[22] S. A. Hughes, Phys Rev D 64, 064004 (2001).

[23] K. Glampedakis, S. A. Hughes, and D. Kennefick, Phys Rev D 66, 064005 (2002).

[24] L. Barack and C. Cutler, Phys Rev D 69, 082005 (2004).

[25] N. Sago, T. Tanaka, W. Hikida, and H. Nakano, Prog Theor Phys 114, 509 (2005).

[26] N. Sago, T. Tanaka, W. Hikida, K. Ganz, and H. Nakano, Prog Theor Phys 115, 873 (2006).

[27] J. R. Gair and K. Glampedakis, Phys Rev D 73, 064037 (2006).

[28] E. Barausse, S. A. Hughes, and L. Rezzolla, Phys Rev D 76, 044007 (2007), 0704.0138.

[29] K. Ganz, W. Hikida, H. Nakano, N. Sago, and T. Tanaka, Prog Theor Phys 117, 1041 (2007).

[30] . . Flanagan and T. Hinderer, Phys Rev D 75, 124007 (2007).

[31] S. Babak, H. Fang, J. R. Gair, K. Glampedakis, and S. A. Hughes, Phys Rev D 75, 024005 (2007).

[32] M. Punturo et al., Classical Quant Grav 27, 084007 (2010).

[33] C. Cutler and M. Vallisneri, Phys Rev D 76, 104018 (2007), 0707.2982.

[34] M. E. Normandin, A. Vajda, and S. R. Valluri, Theor Comput Sci. 409, 241 (2008).

[35] L. Iorio, H. I. M. Lichtengger, M. L. Ruggiero, Ch. Corda, Astrophys Space Sci 331 351-395 (2011)

[36] C. W. F. Everitt el al., Phys Rev Lett 106221101 (2011).

[37] L. Sadeghian and C. M. Will, Classical Quant Grav 28225029 (2011)

[38] L. Iorio, Phys Rev D 84124001 (2011) 
[39] L. Iorio, Mon Not R Astron Soc 411 453-463 (2011)

[40] C. M. Will, Astrophys J Lett 6741 L25-L28 (2008)

[41] P. G. Komorowski, Ph.D. thesis, The University of Western Ontario (2011), http://ir.lib.uwo.ca/etd/115

[42] P. G. Komorowski, S. R. Valluri, and M. Houde, Classical Quant Grav 27, 225023 (2010).

[43] J. W. Mellor, Higher Mathematics for Students of Chemistry and Physics, 4th ed. (Dover Publications Inc., Mineola, New York, 1955).

[44] M. R. Spiegel, Theory and Problems of Advanced Calculus (McGraw-Hill, New York, 1963). 


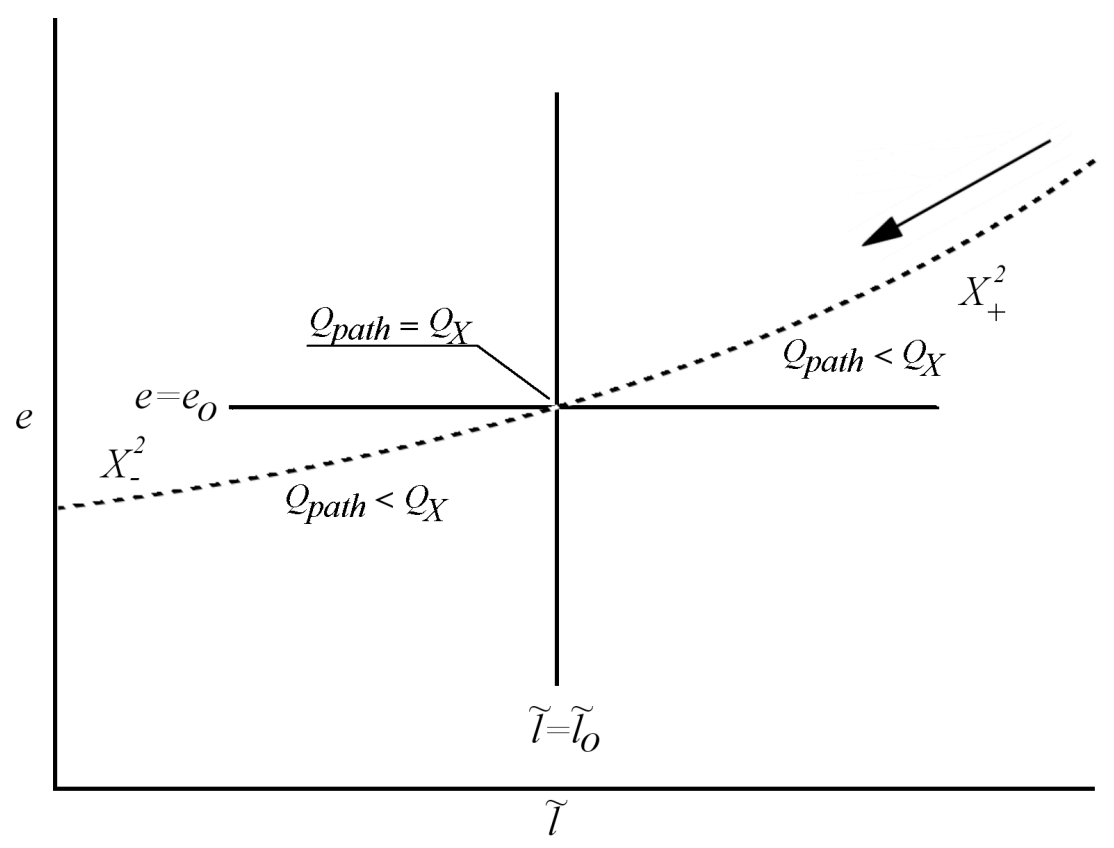

Figure 1: A generic representation of $Q_{\text {path }}$ (short-dashed line) is depicted in the $\tilde{l}-e$ plane as it makes contact of the first order at a point $\left(\tilde{l}_{o}, e_{o}\right)$ on the $Q_{X}$ surface. The direction in which the orbit evolves is shown by the arrow. We offer a generic representation to emphasise that any $Q_{\text {path }}$, which is predicted by a radiation back-reaction model, may be tested in this manner. 